\title{
IL PROBLEMA STORIOGRAFICO DELLA NOZIONE DI "FILOSOFIA SCOLASTICA" E LA GENESI DELLA NOZIONE DI "SECONDA SCOLASTICA"
}

\author{
Marco Forlivesi ${ }^{I}$
}

RIASSUNTO: Il saggio intende delucidare la genesi delle nozioni di "filosofia scolastica" e "seconda scolastica". Le ricerche svolte fino a oggi sulla storia della nozione di "scolastica" non sembrano aver preso in esame la questione della possibile diversità della storia della nozione di "filosofia scolastica" rispetto alla storia delle nozioni di "teologia scolastica" e di "scolastica" tout court. In questo studio viene proposta la tesi secondo la quale la storia della nozione di "filosofia scolastica", benché strettamente connessa con la storia della nozione di "teologia scolastica", non è identica a quest'ultima e vengono presentati elementi e indizi circa i tempi e i modi nei quali, tra prima modernità e inizio del XX secolo, l'aggettivo "scolastica" fu utilizzato per designare un tipo di filosofia che viene ritenuto essere altro dal genere di filosofia praticato dagli autori moderni. I risultati raggiunti forniscono la base per determinare le ragioni che portarono Carlo Giacon a formulare, negli anni ' 40 del XX secolo, la nozione di "seconda scolastica", della quale vengono individuate le originarie finalità e implicazioni teoretiche.

PAROLE CHIAVE: Filosofia scolastica. Seconda scolastica. Neo-scolastica. Carlo Giacon. Storia della storiografia filosofica.

Se questo breve scritto ambisse a una qualche completezza, dovrebbe illustrare innanzi tutto l'origine e l'evoluzione delle nozioni di "scolastica" in generale e di "filosofia scolastica" in particolare. Dato che per affrontare questi temi occorrerebbe una monografia, riserverò al problema storiografico costituito dall'evoluzione della nozione di "filosofia scolastica" solamente alcuni cenni.

Per nostra fortuna, la storia della nozione di scholastica è già stata tracciata dal compianto Riccardo Quinto nel suo magistrale lavoro

1 Dipartimento di Scienze Filosofiche, Pedagogiche ed Economico-Quantitative. Università degli Studi di Chieti e Pescara. http://unich-it.academia.edu/MarcoForlivesi. 
"Scholastica". Storia di un concetto; a esso, pertanto, rinvio in ogni caso il lettore. ${ }^{2}$ Coloro che volessero poi approfondire la conoscenza dei contesti concettuali e storiografici nei quali operarono gli autori esaminati da Quinto, possono ricorrere ai contributi contenuti nei cinque volumi della Storia della storie generali della filosofia, di cui il prof. Piaia è, assieme a Giovanni Santinello, curatore generale. ${ }^{3}$ Dirò dunque ora in breve, come introduzione al tema del presente scritto, che la fortuna della nozione di "scolastica" poggia sul fatto che in essa sono congiunte una chiara base etimologica e un prolungato e multiforme uso storico.

La base etimologica di "scolastica" risiede in un duplice dato. Anteriormente al diffondersi delle lingue vernacole come possibile veicolo di elaborazione e trasmissione di scienza ed erudizione, ossia al tempo in cui gli ambienti e gli ambiti in cui si svolgeva attività d'insegnamento erano detti scholae, l'aggettivo scholasticus era riferito a tutto ciò che concerneva gli istituti di istruzione superiore. A questo va aggiunto che schola poteva avere anche il senso di "schiera" e, in effetti, il termine fu utilizzato, dal tardo medioevo in avanti, anche per indicare insiemi di pensatori uniti dalla condivisione, almeno presunta, di alcune tesi.

Per quanto riguarda gli usi del sostantivo schola e degli aggettivi (e poi aggettivi sostantivati) scholasticus e, successivamente, scholastica, essi hanno conosciuto, tra il XII e il XVII secolo, una costante fluttuazione tra il piano dell'ambito delle istituzioni ove il sapere era trasmesso e il piano dei contenuti di quel sapere. Credo si possa dire che i momenti più significativi nella storia - molto complessa, in verità - del significato di questi termini siano due.

Il primo è costituito dal dibattito sollevato dalle critiche - spesso convergenti ma non coincidenti - mosse da alcuni teologi fin dal XII secolo, dagli humanistae (ossia dai docenti universitari, o più spesso aspiranti tali, di humanae litterae) e da Lutero (almeno nelle prime fasi della Riforma) ai teologi "universitari". È in questo contesto che si forma l'idea per cui la teologia universitaria è caratterizzata da un rapporto peculiare tra la speculazione filosofica e la riflessione sulla rivelazione: un rapporto in forza del quale, nel corso del tempo, da un lato le due attività si distinguono sempre più nettamente, dall'altro si sviluppa sia il controllo - anche per vie politiche - della seconda sulla prima, sia l'influenza della prima sulla seconda.

${ }^{2}$ QUINTO, 2001.

${ }^{3}$ SANTINELLO; PIAIA, 1979-2004 (21993-). 
L'espressione theologia scholastica venne pertanto impiegata per indicare quel tipo di teologia che fu teorizzato in età tardo-medievale e rinascimentale dai teologi cattolici; specialmente, ma non esclusivamente, dai teologi appartenenti alla via antiqua. ${ }^{4}$ Si trattò di un tipo di teologia tutt'altro che monolitico ma che, nel secolo posteriore al Concilio di Trento, fu l'unico ad avere - almeno ufficialmente - diritto di cittadinanza in ambito cattolico.

Il secondo momento significativo nella storia dei termini in esame si colloca nel XVII secolo. In questo secolo si assiste al diffondersi di un nuovo modello di fisica e di scienza, al successo delle accademie come ambiti di discussione scientifica e comunicazione erudita diversi dai tradizionali studia e all'indebolirsi del primato del latino come lingua di trasmissione dell'alta cultura. In questo quadro culturale e politico-culturale, il cui consolidamento ha luogo a partire dall'ultimo quarto del XVII secolo, l'aggettivo scholasticus e il sostantivo scholastica ben si prestarono a indicare - efficacemente, perché in modo del tutto vago - ciò che la nuova cultura non intendeva essere. Divenne da quel momento possibile definire scholastici tutti gli autori di livello universitario, specialmente cattolici, collocati nel passato o - detrattivamente - legati a ciò che, a torto o a ragione, i "nuovi pensatori" ritenevano fosse "il passato"; in tal modo, tutto era pronto affinché gli scholastici divenissero "gli altri”, scholastica divenisse il nebuloso insieme delle loro dottrine e scholae il luogo di un'istruzione inconsistente e inutile, oppure le litigiose correnti in cui quegli scrittori si dividevano.

La complessa storia dei termini scholasticus e scholastica, la genericità dei loro significati, fornirono e tuttora forniscono a questi termini notevolissime "doti" di plasticità semantica e costituiscono precisamente uno dei fondamenti della loro fortuna. Agendo sull'intensione e sull'estensione di queste nozioni, esse possono essere utilizzate per designare le entità più diverse. Scolastici, ad esempio, potrebbero essere detti tutti quei pensatori che furono attivi nell'ambito degli istituti d'istruzione superiore nel lungo periodo in cui il latino fu la lingua dell'alta cultura. Il termine potrebbe però essere utilizzato per indicare gli autori specificamente universitari; oppure per indicare tutti gli autori che operarono nell'ambito degli studia in genere a partire dal tempo in cui nacquero le università; oppure ancora tutti gli autori che operarono in specifici ambiti dell'istruzione universitaria, quali quelli della teologia o della fisica. $\grave{E}$ inoltre possibile tentare di operare passaggi dal campo istituzionale a

\footnotetext{
${ }^{4}$ Sulla genesi delle due contrapposte nozioni di "teologia scolastica" circolanti a partire dalla prima metà del XVI secolo, cf. anche DE HANGEST, 2009.
} 
quello dottrinale e denominare "scolastici" quegli autori che siano fautori veri o presunti tali - di un certo insieme di tesi.

Stanti queste premesse, non stupirà che i tentativi di definire positivamente scholastici e scholastica portino facilmente ad esiti paradossali. Si supponga, ad esempio, di attribuire la qualifica di scholastici a quei filosofi che siano stati assertori, tra tardo medioevo e prima età moderna, di una fisica di tipo qualitativo. Se così facessimo, Marsilio Ficino e Francis Bacon dovrebbero essere detti scholastici; si potrebbe dubitare, invece, che Jean Buridan e Thomas Bradwardine possano essere detti tali. Ci si dovrebbe anche chiedere, peraltro, se e quando una definizione siffatta sia stata proposta. Si supponga allora di attribuire la qualifica di scholastici a quei teologi che abbiano sostenuto l'ancillarità della fisica e della metafisica nei confronti della teologia dogmatica. Se così si facesse, ne verrebbe che tutti gli autori della via moderna, da Guglielmo di Ockham fino a Gabriel Biel, potrebbero difficilmente essere detti scholastici; forse ciò potrebbe rallegrare qualche antico neo-tomista, ma va contro la storia dell'uso del termine.

Degni di nota, a proposito della problematicità dei termini in questione, mi sembrano i casi, a cavallo tra la fine del XVI secolo e la prima metà del XVII, di Cesare Cremonini e di René Descartes. Il primo fu un autore universitario per eccellenza, un perfetto esempio di aristotelico rinascimentale, il quale riteneva che il proprio compito di docente di filosofia naturale consistesse nel fornire un'interpretazione dei testi dello Stagirita che ne mostrasse la coerenza e profondità; il secondo fu il noto fautore di una metafisica e di una fisica di matrice sostanzialmente lulliana, che incorporava nel proprio pensiero le speranze tecnologico-sociali di Francis Bacon e la matematizzazione della fisica operata da Galileo Galilei. Ebbene, nelle opere pubblicate da Descartes non si parla di scholastici o di scholastica; si parla - peraltro raramente - di insegnamenti o concetti in uso in scholis. Il senso di ciò che Descartes dice è chiaro: allorché parla di scholae, parla di ciò che veniva insegnato ai suoi tempi a livello universitario; certamente, almeno nelle opere che volle rendere pubbliche, non utilizza i termini summenzionati per delineare un preciso tipo di filosofia o una concezione definita del rapporto tra filosofia e teologia dogmatica. Parallelamente, si può osservare che neppure Cremonini - il più celebre docente universitario dei suoi tempi, come ebbe a dire un suo biografo - presentava sé stesso come un filosofo scolastico; scholasticus ha piuttosto, 
nei suoi scritti, o il semplice senso di "concernente le scholae", o il senso di "relativo agli atti scolastici".

Poiché nei ricchissimi volumi della Storia delle storie generali della filosofia la poliedricità della nozione di scolastica emerge con chiarezza, mi permetto di rinviare il lettore a essi. ${ }^{6}$ In quell'opera, assieme a una compiuta presentazione del modo in cui la storia della filosofia medievale fu concettualizzata dai pensatori dal XVI al XIX secolo, il lettore troverà numerosissime menzioni dei termini scholastica e scholastici (e dei loro derivati) e, con esse, l'ampia gamma di modi in cui questi termini furono intesi. Credo che, dopo una tale lettura, chiunque potrà dire della scholastica ciò che il malvagio Olinto, ne Il Demetrio del Metastasio, dice a proposito della fedeltà tra gli amanti: «come l'araba fenice, che vi sia ciascun lo dice, dove sia nessun lo sa».

Se l'indagine storiografica mostra la problematicità della nozione di scholastica, ciò non implica che non sia possibile tracciarne una storia. Tra la mole di materiali offerti dalla Storia delle storie generali della filosofia e dal già ricordato volume di Riccardo Quinto, e aggiungendo forse qualche notizia a quelle che queste opere forniscono, mi sembra al presente utile isolare una linea specifica: quella lungo la quale furono elaborate quelle concezioni di "scolastica" in generale e di "filosofia scolastica" in particolare che furono proprie della filosofia e della storiografia neo-scolastiche.

In effetti, mi sembra che il sorgere della nozione di filosofia scolastica necessiti ancora di qualche ricerca. ${ }^{7}$ Nell'enciclica Aeterni Patris, resa pubblica

${ }^{5}$ Cf. ad esempio CREMONINO, 1990, p. 151-226. La commedia Le nubi - un capolavoro che attende un moderno e capace curatore - è un attacco a Giorgio Raguseo e, più in generale, a quegli autori universitari, anche tardo-medievali, che, secondo Cremonini, non avevano posto al centro della propria riflessione la corretta interpretazione di Aristotele.

${ }^{6} \mathrm{Si}$ ha l'impressione che l'opera sia nota anche ai lettori non italiani molto più di quanto sia menzionata. Si potrebbe dire che l'onestà intellettuale non sia tra i beni che più abbondano su questa Terra e che essa sia comunque equamente distribuita, così che non sembra che se ne possa trovare in ambito anglosassone o tedesco più di quanta se ne veda in Italia. Credo sia giusto ricambiare la cortesia passando qui sotto silenzio quegli scritti, non rari ma quasi sempre scarsamente significativi, che "dimenticano" l'opera del gruppo di lavoro inaugurato da Giovanni Santinello.

${ }^{7}$ L'unico scrittore anteriore al XIX secolo che QUINTO, 2001, esplicitamente indica come autore di una riflessione specificamente dedicata alla nozione di "filosofia scolastica" è Jean Mabillon. Per l'esattezza, Quinto fa riferimento a MABILLON, 1691, partie 2, chap. 9-10, p. 242-267. Nel testo in questione, però, Mabillon non opera una saldatura tra le nozioni di "filosofia" e di "scolastica". In esso fa uso, sì, dell'espressione "auteur scolastique" e vi difende un insegnamento della filosofia ben ordinato, sotto la guida di un maestro, che prepari agli studi teologici; ciononostante, i contenuti di quell'insegnamento né sono definiti da Mabillon "filosofia scolastica", né hanno granché a che fare con la filosofia universitaria (in senso lato) medievale o rinascimentale. Peraltro, Mabillon non 
a Roma da papa Leone XIII il 4 agosto 1879, viene fatto un esplicito riferimento alla bolla Triumphantis Hierusalem promulgata da papa Sisto V il 14 marzo 1588. In quell'atto, con il quale Sisto V proclamava Bonaventura da Bagnoregio dottore della Chiesa, si affermava il valore della theologia scholastica e la necessità di coltivarla e difenderla.

Divino [...] illius munere, qui solus dat spiritum scientiae et sapientiae et intellectus, quique Ecclesiam suam per saeculorum aetates, prout opus est, novis beneficiis auget, novis praesidiis instruit, inventa est a maioribus nostris sapientissimis viris theologia scholastica [...]. Et huius quidem tam salutaris scientiae cognitio et exercitatio, quae ab uberrimis divinarum litterarum, summorum pontificum, sanctorum patrum et conciliorum fontibus dimanat, semper certe maximum Ecclesiae adiumentum afferre potuit, sive ad Scripturas ipsas vere et sane intelligendas et interpretandas, sive ad patres securius et utilius perlegendos et explicandos sive ad varios errores et haereses detegendas et refellendas. [...] Et profecto rem ita se habere ipsimet veritatis inimici sunt iudices, quibus theologia scholastica maxime est formidolosa, qui profecto intelligunt, apta illa et inter se nexa rerum et causarum cohaerentia, illo ordine et dispositione, tamquam militum in pugnando instructione, illis dilucidis definitiones et distinctiones, illis argumentorum firmitate et acutissimis disputationibus lucem a tenebris, verum a falso distingui, eorumque mendacia multis praestigiis et fallaciis involuta, tamquam veste detracta patefieri et denudari. Quanto igitur magis illi hanc munitissimam scholasticae theologiae arcem oppugnare et evertere conantur, tanto magis nos decet hoc invictum fidei propugnaculum, et haereditatem patrum nostrorum conservare et tueri $[\ldots] .^{8}$

Nella enciclica Aeterni Patris, il comando sistino viene interpretato come riguardante anche la filosofia.

Quae verba, quamvis theologiam scholasticam dumtaxat complecti videantur, tamen esse quoque de philosophia eiusque laudibus accipienda perspicitur. ${ }^{9}$

sembra apprezzare il discours scolastique e se si cerca nell'indice dei soggetti il termine Scolastique si viene rinviati puramente e semplicemente a Theologie scolastique. Per queste ragioni, credo che non si possa includere Mabillon né tra i teorizzatori di una precisa nozione di "filosofia scolastica", né tra gli utilizzatori di una nozione siffatta.

${ }^{8}$ SIXTUS V, 1588 (TOMASSETTI, 1863, p. 1005b-1012b, in part. \$10, p. 1009a-1010a). Dal testo della bolla non si riesce a inferire indubitabilmente se la theologia scholastica venga considerata identica alla sacra theologia o sia considerata un tipo di sacra theologia; entrambe le letture sono possibili.

${ }^{9}$ LEO XIII, 1879, p. 107. 
Tra il tempo di Sisto V e il tempo di Leone XIII ha dunque avuto luogo un mutamento sia nella concezione della teologia rivelata, sia nella concezione della filosofia. Nella bolla di Sisto V la theologia scholastica appare essere una teologia rivelata sistematica, il cui ordine e la cui efficacia argomentativa dipendono certo anche da un qualche utilizzo della filosofia, tuttavia quest'ultima non è mai esplicitamente nominata e considerata indipendentemente dalla prima. Non per nulla, ancora nella produzione degli autori universitari (prendendo il termine in senso lato) della prima metà del Seicento, le questioni filosofiche più complesse sono trattate nel contesto delle opere teologiche.

Per quanto mi è noto, la prima opera di vasto impegno che rechi nel titolo l'espressione philosophia scholastica è la Universa philosophia scholastica del gesuita Richard Lynch, pubblicata in tre volumi a Lione nel 1654. ${ }^{10}$ Nell'opera di Lynch non trovo però alcuna esplicita tematizzazione dell'aggettivo scholastica, tanto da indurmi a pensare che il titolo potrebbe essere semplicemente tradotto con la parafrasi "Corso completo di filosofia universitaria”. Nel primo volume della successiva, e postuma, Universa theologia scholastica del medesimo autore si parla, dando all'espressione un senso positivo, di scholastica acumina; anche in questo caso, tuttavia, ciò che è in questione è un tipo di teologia, ossia la theologia scholastica. ${ }^{11}$ Questo non significa che Lynch non fornisca una rappresentazione precisa della sua philosophia: essa, scrive, è opera della ragione, tale da avvantaggiarsi in qualche modo della tradizione e della teologia, asciutta nello stile. ${ }^{12}$ Manca, però, un collegamento tematico tra questo tipo di filosofia e l'aggettivo scholasticus e tutto ciò che resta a questo proposito è il titolo dell'opera.

La seconda metà del XVII secolo non sembra presentare, in ambito cattolico, mutamenti significativi, ma solo variabili e graduali mutamenti di accento. Nel Cursus philosophicus, sive Philosophia scholastica, ad mentem Scoti,

\footnotetext{
${ }^{10}$ Segnalo che, in qualche modo, si potrebbe forse risalire indietro nel tempo di altri vent'anni. Nel 1636 il teatino Zaccaria Pasqualigo pubblica a Roma (con approvazioni di stampa del 1632 e 1633) il secondo volume, e seconda parte, delle sue Disputationes metaphysicae. Ebbene, questa parte reca il seguente titolo: Pars secunda in qua de passionibus obiecti primae philosophiae disseritur, et principia scholasticae doctrinae stabiliuntur. È chiaro che la doctrina scholastica in questione è una dottrina di natura filosofica, tuttavia Pasqualigo non spiega cosa intenda con l'aggettivo scholasticus.

${ }^{11}$ LINCAEUS, 1679 , c. (carta/pagina non numerata; si indica la segnatura) D6v.

${ }^{12}$ LYNCEUS, 1654 , v. 1, c. (carta/pagina non numerata; si indica la segnatura) a6r. Lynch, però, non spiega in cosa esattamente consista la relazione che, a suo avviso, deve sussistere «[...] inter scientias humanas, et scientiam divinam, theologiamve».
} 
nova et congruentiori addiscentibus methodo disposita del francescano osservante Tomás de Llamazares, pubblicato nel 1670, l'espressione philosophia scholastica non è oggetto di alcuna specifica riflessione e appare confinata al titolo del volume. Nella prefazione al testo Llamazares parla, invece, di philosophia Aristotelica e di theologia scholastica, presentando la prima come preliminare allo studio della seconda. ${ }^{13}$ Invero, segnalo che nella approbatio del volume di Llamazares a firma del francescano osservante José Jiménez Samaniego l'espressione philosophia scholastica compare due volte e appare essere un insieme di dottrine in qualche modo definite; neppure Jiménez Samaniego, tuttavia, si sofferma a fornire spiegazioni. ${ }^{14}$

Nel 1671 il domenicano Serafino Piccinardi, a quel tempo già docente di metafisica in via Thomae presso lo Studio pubblico di Padova, pubblica il primo volume della propria Philosophia dogmatica peripatetica Christiana in patrocinium Aristotelis ac in osores eiusdem. In questo testo Piccinardi scrive che la «philosophia decretoria, seu peripatetica adhuc bene dividitur in dogmaticam circa dogmata", che sono i principi primi di una scienza, "et scholasticam circa theses", ossia le conclusioni di quella scienza. ${ }^{15}$ In questo contesto, tuttavia, l'aggettivo scholasticus sembra far riferimento più all'ordine secondo il quale deve essere strutturata una filosofia ben costruita che ai contenuti della filosofia vera; quei contenuti, invece, sono espressi dall'aggettivo peripatetica. L'opera di Piccinardi mira precisamente a questo obiettivo: mostrare che la filosofia che il cristiano deve professare è quella di Aristotele, specificamente nella forma che essa ha assunto grazie all'opera "purificatrice" compiuta su di essa da Tommaso d'Aquino.

Piccinardi non fu un autore isolato; anzi, ben presto la sua divenne una posizione pressoché obbligata in ambito cattolico. Il 2 dicembre 1673 il Sant'Uffizio inviò una circolare a tutti gli inquisitori con la quale si proibiva di stampare testi nei quali si negasse che le sostanze composte fossero costituite da

\footnotetext{
${ }^{13}$ LLAMAZARES, 1670, Ad omnes philosophiae et veritatis studiosos auctoris praefatio, in part. c. (carta/ pagina non numerata; si indica la segnatura) ã4r e ã $4 v$. A c. (carta/pagina non numerata; si indica la segnatura) é $2 v$ del medesimo testo compaiono anche il sostantivo scholastici e l'espressione ad scholae stylum, ma non mi sembra che la prospettiva di Llamazares sia diversa da quella che era stata di Lynch. Si può anche osservare che Llamazares non presta alcuna attenzione agli autori "moderni": a c. (carta/pagina non numerata; si indica la segnatura) é $2 r$ scrive che «[...] in Physicis nullo duce praeter Aristotelem indigemus». Una parziale eccezione a questa dichiarazione può essere rintracciata nelle pagine conclusive della parte del volume dedicata alla fisica, ove l'autore abbraccia la teoria dello impetus prendendo posizione, in modo non polemico ma esplicito, contro Aristotele.

${ }^{14}$ LLAMAZARES, 1670, c. (carta/pagina non numerata; si indica la segnatura) é4r.

${ }^{15} \mathrm{Il}$ passo del testo di Piccinardi è riportato in POPPI, 2001, p. 105-107, nota 10.
} 
materia e forma; ${ }^{16}$ veniva con ciò indirettamente ma fermamente ribadito che la philosophia peripatetica doveva essere la filosofia d'elezione per il cristiano cattolico. Precisamente questo, peraltro, è ciò che sostiene il gesuita Giovanni Battista De Benedictis nella sua Philosophia peripatetica, stampata in quattro volumi dal 1687 al 1692, e nelle Lettere apologetiche in difesa della teologia scolastica e della filosofia peripatetica, stampate nel 1694. Se, però, a proposito della prima opera si può semplicemente dire che l'espressione scholastica philosophia non vi compare e in essa non si danno casi in cui l'aggettivo scholasticus sia riferito alla filosofia, la seconda opera presenta una terminologia più complessa. In essa compaiono i sintagmi "scolastica", "teologia scolastica", "scolastici" e "scuola" e viene addirittura riportato il medesimo passo della bolla Triumphantis Ierusalem che sarà riportato nella Aeterni Patris. Da quel passo, però, De Benedictis non deduce che ciò di cui quella bolla parla sia anche una forma di filosofia; al contrario, questo autore propugna il valore esattamente di ciò di cui la bolla di Sisto V effettivamente parla, ossia una forma di teologia rivelata la quale fa uso della filosofia per i propri scopi. ${ }^{17} \mathrm{E}$ vero che, almeno in un luogo dell'opera, il sostantivo "scolastica" è chiaramente riferito alla filosofia; nondimeno, "scolastica" non appare essere specificamente quella forma di filosofia che è propizia alla teologia e che quest'ultima in qualche misura utilizza; essa appare essere, invece, l'“alta cultura" in generale. ${ }^{18}$ La filosofia che, come espressione della ragione, può e deve essere utilizzata dalla teologia è, per De Benedictis, la filosofia peripatetica. ${ }^{19}$

${ }^{16}$ Il testo della circolare è riportato in DONATO, 2009, p. 603.

${ }^{17}$ DE BENEDICTIS, 1694, p. 14-15.

18 «Non è ingiuria, no, è ossequio alla Divinità, che parla, metterle a piedi la filosofia, e la natura, che confessino, dircisi dal Signore molte cose superiori, ma non già veruna contraria al lume impressoci nella mente. So, che molti degli antichi Padri giudicarono pernicioso un tale accoppiamento: ma parlavano della scolastica, che regnava in que' tempi: parlavano della filosofia, che superba vuole insegnare alla maestra, e far regola al credere [...]; e non di quella, che umile si accosta per apprendere ciò che non sa, e correggere gli errori della natia debolezza col magistero infallibile della fede» (DE BENEDICTIS, 1694, p. 25). Si consideri anche l'uso che De Benedictis fa del termine "scuola"; cf., per esempio, DE BENEDICTIS, 1694, p. 106.

19 «<Aristotele> Vede i principii della sua filosofia, le sue massime, i suoi discorsi trapiantati nella teologia per servire di tronco alle verità della fede, e divenir con esse per mirabile innesto una cosa medesima» (DE BENEDICTIS, 1694, p. 59). De Benedictis chiarisce tuttavia presto che quel peripatetismo che la divina provvidenza ha salvaguardato al fine «[...] di accordare la ragione con la fede, e dar metodo, e forma alle scuole de' suoi teologi» (DE BENEDICTIS, 1694, p. 82) è quella che è stata "[...] resa da' teologi mansueta, e ossequiosa, si accorda in ogni punto colla religione, ed ascolta la fede come maestra» (DE BENEDICTIS, 1694, p. 76). 
Un testo modesto ma di grande interesse per la questione qui in esame è la Summa philosophiae scholasticae et Scotisticae del francescano osservante Sebastien Dupasquier. Pubblicata nel 1691 o 1692 a Lione, essa contiene un interessante prologo di argomento metodologico e didattico nel quale l'autore illustra le ragioni del titolo dell'opera. Dupasquier è al corrente di alcune delle "novità" circolanti al suo tempo in ambito filosofico e afferma esplicitamente di non volerne tenere conto: «Abstinui a novitatibus Cartesianis, Gassendianis et similibus, quia Scotus a novitatibus abhorruit, Aristotelem et solidam maturamque secuutus antiquitatem $» .{ }^{20} \mathrm{Ci}$ si aspetterebbe dunque di incontrare la formulazione di un'esplicita opposizione tra la philosophia scholastica e quella dei recentiores. Ecco, invece, ciò che scrive Dupasquier.

Quad titulum, prorsus arbitrarius est et absque peculiari consilio. 1. Dixi summam philosophiae non quod non sit simplex compedium, sed quia partes et tractatus omnes philosopho religioso et ad theologiam aspiranti necessario complectitur. 2. Scotisticam, quia quantum potui adhaesi Subtilissimi Doctoris menti [...]. 3. Addidi scholasticam quia curavi semper in forma argumentativa procedere, tum in asserendo et probando, tum in opponendo, tum in respondendo: nam scribo tironibus, ut eos ad argumentandum formem, non magistris, qui hoc non egent adjumento. ${ }^{21}$

Come si vede, stando alle dichiarazioni dell'autore, la scolasticità - se così posso dire - dell'opera risiede non nel fatto che essa si opponga, non curandosi di loro, ai moderni, bensì nella sua struttura didattica.

Se gli autori cattolici della seconda metà del Seicento non sembrano inclini a tematizzare e utilizzare l'espressione philosophia scholastica per designare un tipo definito di filosofia, l'espressione è invece presente nelle opere di influenti autori protestanti almeno a partire dal 1688. Nella prima edizione del suo Polyhistor sive de notitia auctorum et rerum commentarii, stampata per l'appunto in quell'anno, Daniel Georg Morhof, presentando la storia dell'università di Oxford pubblicata nel 1674 da Antony Wood, scrive che questo autore "cum diligentissime recenset antiquissimae Academiae Oxoniensis originem et progressum, operosus est in theologiae et philosophiae scholasticae historia percensenda». Morhof, tuttavia, non fornisce alcuna definizione di philosophia scholastica. Si potrebbe pensare che il sintagma, nel

${ }^{20}$ DUPASQUIER, 1692, v. 1, Ad philosophiae Scotisticae candidatos, c. (carta/pagina non numerata; si indica la segnatura) aa3 $v$.

${ }^{21}$ DUPASQUIER, 1692, v. 1, c. (carta/pagina non numerata; si indica la segnatura) aa3r. 
contesto ora menzionato, significhi semplicemente "filosofia universitaria"; considerati però i contenuti dell'opera di Wood, l'espressione di Morhof potrebbe effettivamente in qualche misura connotare i contenuti della filosofia che veniva insegnata in quella università. ${ }^{22}$

Più esplicito nel mettere a tema la nozione e l'espressione "filosofia scolastica" è invece Christian Thomasius. Nella sua Introductio ad philosophiam aulicam seu primae lineae libri de prudentia cogitandi atque ratiocinandi ubi ostenditur media inter praejudicia Cartesianorum, et ineptias peripateticorum, veritatem inveniendi viam, pubblicata anch'essa nel 1688, così scrive.

\$61. Interea Christianus orbis inter densissimas tenebras agebat, et circa nonum seculum tam indocta erant tempora, ut qui philosophiae operam navabat, vulgo magus haberetur. Tandem exorti illi, quod hodieque scholasticos appellamus, qui, cum initio theologiae in coenobiis operam navarent, mox toti ad philosophiam abrepti eam supra theologiam extulerunt, quare potius philosophis quam theologis annumerari meruerunt. \$62. Primum ad scholasticam philosophiam gradum in occidente videtur struxisse Johannes Rucelinus Britannus... ${ }^{23}$

Nel ben più celebre Dictionaire historique et critique dell'ugonotto Pierre Bayle, pubblicato nel 1697, non è presente una voce Scholastique, tuttavia l'opera contiene decine di occorrenze dei termini della famiglia che fa capo all'aggettivo scholastique; in particolare, si danno anche alcune occorrenze dell'espressione philosophie scholastique (quasi sempre, tuttavia, nella forma philosophie et theologie scholastique) e almeno un'occorrenza dell'espressione philosophe scholastique. ${ }^{24}$ Probabilmente a causa della superficialità della mia lettura del testo, non ho trovato nell'opera una tesi storiografica circa la filosofia scolastica quale quella formulata da Christian Thomasius. Nondimeno, i contesti nei quali l'aggettivo e il sostantivo scholastique compaiono non lasciano dubbi sul significato che Bayle assegna a questi ultimi: nel Dictionaire essi denotano o connotano un ambito universitario popolato di litigiosi autori frequentemente in conflitto tra loro, una pratica intellettuale caratterizzata da dispute inutili e vuote sottigliezze, una teologia spiritualmente sterile figlia tanto di quella pratica quanto di una vacua filosofia di derivazione aristotelica, e, infine, una filosofia labirintica, intessuta di chimere e oscurità,

${ }^{22}$ MORHOF, 1688, p. 188. Per quanto sono stato capace di vedere, Wood non utilizza l'espressione.

${ }^{23}$ THOMASIUS, $1688, \$ \$ 61-70$, p. $30-33$, testo a p. 30.

${ }^{24}$ BAYLE, 1740 , v. 2, p. 459. 
linguisticamente rozza, nemica della religione e dalla quale molti recenti filosofi hanno preso le distanze. ${ }^{25}$ Gli scholastiques non sono confinati da Bayle al medioevo; al contrario, è chiaro che egli ritiene che ve ne siano molti anche nel secolo in cui egli vive. In almeno un'occasione, riferendosi esplicitamente a Serafino Piccinardi, fa uso dell'espressione scholastiques modernes. ${ }^{26}$ Anche gli «scholastiques espagnols», nei confronti dei quali esprime un invariabile biasimo, appaiono essere autori a lui contemporanei. ${ }^{27} \mathrm{~A}$ rigori, Bayle non identifica filosofia scolastica e peripatetismo, come si vede nella voce che dedica ad Andrea Cesalpino, tuttavia scrive anche che «[...] la scholastique [...] retient la plûpart des Péripatéticiens». ${ }^{28}$ Sembrerebbe, invece, che tutti coloro che egli denomina scolastici siano autori cattolici; non mancano però voci, quali quelle dedicate a John Knox, Gijsbert Voet o Christoph Scheibler, nelle quali segnala la presenza di dottrine e metodi "scolastici" anche in questi scrittori non cattolici, pur tentando poi di mettere in luce la presenza in essi anche di tratti intellettuali positivi.

Con erudizione più ampia di quella di Thomasius e con sistematicità più forte di quella di Bayle, la nozione di "filosofia scolastica" è messa a tema e utilizzata nella fortunatissima Historia critica philosophiae di Jacob Brucker, pubblicata in prima edizione dal 1742 al 1744 . Nel terzo tomo di quest'opera, la sezione intitolata De scholasticis presenta esplicitamente e coscientemente gli scholastici nei termini di una corrente filosofica. ${ }^{29}$ Dopo aver delineato quelle che, a suo avviso, sono le cause e le origini della scolastica nell'alto medioevo e, specificamente, la centralità in essa dapprima della dialettica e successivamente della metafisica, Brucker, fondendo le consuete critiche alla "scolastica" e la tesi storiografica di Thomasius, scrive quanto segue.

${ }^{25}$ Piuttosto che fornire una troppo lunga lista di riferimenti, ritengo utile segnalare al lettore lo strumento attraverso il quale rilevare con facilità e immediatezza quanto ho ora sostenuto. Si tratta dell'edizione elettronica dell'edizione del 1740 del Dictionaire realizzata nel contesto del Project for American and French Research on the Treasury of the French Language e accessibile all'indirizzo internet artfl-project.uchicago.edu/node/60 (indirizzo consultato il 13 settembre 2014). Il lettore potrà prendere visione da sé delle decine di occorrenze dei termini scholastique e derivati e dei contesti nei quali essi compaiono.

${ }^{26}$ BAYLE, 1740 , v. 1, p. 328.

${ }^{27}$ Cf., ad esempio, BAYLE, 1740, v. 4, p. 174.

${ }^{28}$ BAYLE, 1740 , v. 1, p. 239. Bayle trae il testo che ho ora riportato dall'opera La vie de monsieur Descartes di Adrien Baillet, pubblicata nel 1691.

${ }^{29}$ BRUCKERUS, 1743, periodus 2, pars 2, lib. 2, cap. 3, sect. 2, in particolare $\$ \$ 1-2$, p. 709-730. 
Cumque, qui hoc modo philosophabantur aut monachi essent, aut clerici, tantum non omnes autem theologi, translata haec philosophia ad theologiam est, ipsique divinioris doctrinae professores non tam ancillantem theologiae quam dominantem philosophiam sectariam, qualem supra depinximus, retulere ad eum scopum, cui eo tempore omnis disciplina sacra inserviebat, nempe ut hierarchia ecclesiastica cum capite suo, et dogmatum, quod eam alebat et sustentabat, systemate sarta conservaretur atque tecta. Huic fini egregie inserviebat philosophia haec scholastica, quae Aristoteli male intellecto revelationis mysteria subiiciens, de eorum sensu iuxta illius praecepta disputabat, et infinitis questionum et dubitationum anfractibus more vere sceptico longum ratiociniorum ipsa subtilitate sua incertorum syrma post se trahebat. Quod philosophiae et theologiae genus ubi semel innotuisset, et a summis rerum sacrarum praesulibus, quorum potestati fulcrum exhibebat firmissimum, summo esset favore exceptum, sustentatum, ornatum, totum fere orbem christianum fecit scholasticum, re vera et doctrina et fide et moribus barbarum. [...] Nos pretermissis ceteris, hoc unum notamus, negari non posse, philosophos, et qui re ipsa coincidunt, theologos scholasticos, eo nomine, quamvis subtilissima essent ingenii acie praediti, pro veris philosophis haberi non posse, quod erepta libertate philosophandi omni servire curiae Romanae et ad eius palatum totam philosophiam suam componere coacti fuerint. ${ }^{30}$

Nelle pagine seguenti, Brucker rifiuta l'identificazione di filosofia scolastica e peripatetismo; a suo avviso, con filosofia scolastica si deve intendere un fenomeno più ampio, ossia quella parte della filosofia medievale - che comprende sì l'aristotelismo scolastico, ma non si esaurisce in esso - che è caratterizzata da un certo stile e da un certo scopo: il disputare vanamente e il sostenere il potere politico del clero. Egli ammette anche che una qualche differenza tra filosofia scolastica e teologia scolastica si dà, tuttavia ribadisce l'identità di fatto tra le due. ${ }^{31}$

Ben prima della Aeterni Patris, dunque, Brucker già concettualizza la nozione di "filosofia scolastica" come designante un tipo di filosofia al contempo distinta da ciò che è designato dalla nozione di "teologia scolastica" ma strettamente congiunta ad essa. Inutile dire che Brucker formula tanto sulla prima che sulla seconda un giudizio di valore ben diverso da quello che

${ }^{30}$ BRUCKERUS, 1743, p. 712-713.

31 BRUCKERUS, 1743, p. 718: «Quod porro inter theologiam et philosophiam scholasticam nonnulli distinguunt, recte quidem factum est, parum autem ad quaestionis, de qua $<$ nunc $>$ agitur, solutionem confert; cum non tantum sororio utraque inter se vinculo cohaereat, sed utriusque iidem natales, eaedemque fuerint origines». 
si ritroverà nella Aeterni Patris. Commentando un passo di Jakob Thomasius dedicato ai "vizi" della teologia scolastica, Brucker così scrive.

Pertinere haec non tam ad philosophiam, quam ad theologiam scholasticam, non negamus, sed cum ea quae communem naturam habent, inter se communia quoque attributa habeant, demtis iis, quae ad solam theologiam pertinent, vultus philosophiae scholasticae ex his facile detegi possunt, praecipur si non ad conclusiones tantum, sive quaestiones otiosas inutiles, inepta subtilitate molestas, et vesana curiositates detestabiles, sed ad ipsa quoque ratiocinandi principia, ex suis fontibus non deducta, auctoritatis vero praeiudicio subnixa, et ex gentili Stoicorum et Aristotelicorum dialectica et metaphysica hausta, tum ad ipsam ratiocinandi et concludendi rationem, vagam, incertam, ex utraque parte infinitis ambagibus disputantem, ad notiones, quae adhibitae sunt obscuras, vagas, temerarias, nimiamque mentis praecisione nihil significantes, quaeque reliqua sunt otiosorum hominum deliria respiciatur. ${ }^{32}$

Il Settecento offre anche in ambito cattolico una forte e precisa concettualizzazione della nozione di "filosofia scolastica". La si rinviene nelle opere del benedettino bavarese Veremund Gufl, di cui si prenderà qui in esame il testo più sistematico: la Philosophia scholastica universa principiis s. Thomae d. a. apprime conformata, et contra neotericos praecipue defensa, experimentorum quoque et matheseos tum purae tum mixtae accessionibus aucta, pubblicata in quattro volumi a Ratisbona nel 1750. Il lavoro di Gufl è tanto degno d'interesse quanto poco noto. Esso si apre con una Dissertatio de selectu philosophiae di oltre un centinaio di dense pagine divisa in tre parti: De selectu veri et variis philosophorum sectis, De philosophia scholastica e De tribus scholis. A questo testo va accostata, per esplicita indicazione dello stesso Gufl, l'articolo 11 del trattato 2 della sua metafisica, contenuta nel secondo tomo dell'opera; un articolo dedicato al tema De nexu philosophiae et theologiae atque de Ecclesiae circa naturalia judicio.

Gufl sviluppail proprio pensiero sulla base di cinque punti di riferimento: i dogmi - in senso lato - della fede cattolica, le tradizioni dottrinali cattoliche elaborate in ambito universitario (le scholae), le critiche di matrice umanistica a quelle tradizioni, le critiche di matrice protestante a quelle tradizioni, le

${ }^{32}$ BRUCKERUS, 1743, p. 721. Il testo che Brucker commenta è THOMASIUS, [1693?], p. 228-229. Curiosamente, il passo di Brucker è sorprendentemente prossimo a un passaggio della Aeterni Patris su cui ci soffermeremo; in quel caso, però, coloro che tutto trascinano nella disputa, e dunque nel dubbio, saranno solamente autori posteriori a Tommaso d'Aquino. 
dottrine dei pensatori moderni (i neotherici). Al centro della sua proposta vi è ciò che Gufl denomina esplicitamente e tematicamente "systema philosophiae scholasticae". Il systema philosophiae scholasticae è fondato sulle verità rese manifeste dalla fede e dal lume naturale e su una precisa serie di tesi (che Gufl elenca) tratte da Aristotele. Questo sistema, sostiene Gufl, è stato formulato nel corso di molti secoli grazie a un lungo lavoro di studio e a un attento esame dei suoi contenuti ad opera delle autorità ecclesiastiche cattoliche e di pensatori eminenti. Per la precisione, esso è il commune systema scholasticae philosophiae, ossia il sistema delle verità su cui tutte le scholae concordano. Il processo che porta alla sua formazione è tale per cui le doctrinae scholasticae da cui è costituito "certitudinem quamdam (moralem vocant) in nobis pariant, nihil in communi systemate scholasticae philosophiae contineri, quod veritatibus revelatis adversetur, earumve sinceritatem corrumpere valeat». ${ }^{33}$

L'utilizzo, in esso, di dottrine originariamente proposte da autori pagani, e in particolare da Aristotele, è lecito e opportuno, giacché da un lato quelle dottrine riguardano materie che sono oggetto anche della fede, dall'altro esse sono state vagliate, riformulate, ampliate e rafforzate sulla base delle verità rese note precisamente dalla fede cristiana. Esso non è stato per nulla, come scrivono i detrattori della scholastica, elaborato in età infelici ed oscure; al contrario, è opera di uomini sapientissimi, che gli autori moderni denigrano senza averne letto le opere. Vi sono stati sì abusi nell'uso della filosofia in teologia ed ineleganze, ma i primi possono e devono essere evitati e le seconde sono state corrette.

Le scholae degne di nota sono tre, ossia la scuola tomista, quella scotista $^{34}$ e quella dei recentiores (ossia, sostanzialmente, la scuola gesuitica); la scuola nominalista, invece, non va considerata, in quanto condannata dalla Chiesa. Le scoperte e le dottrine dei neotherici nell'ambito della matematica e della fisica matematizzata e sperimentale non inficiano in nulla il systema philosophiae scholasticae, poiché esso non si fonda né sulla matematica, né su evidenze sperimentali diverse dall'esperienza comune. Al contrario, il systema philosophiae scholasticae fornisce gli strumenti per una fondazione propriamente filosofica di quelle scoperte. Il benedettino bavarese offre anche un esame sintetico ma puntuale e ben informato del pensiero degli autori moderni da Descartes a Berkeley e La Mettrie e degli errori, come egli li qualifica, nei quali

\footnotetext{
${ }^{33}$ GUFL, 1750, v. 1, p. (prima serie) 63.

${ }^{34}$ Per la conoscenza della scuola scotista Gufl fa riferimento principalmente, se non addirittura esclusivamente, a Bartolomeo Mastri.
} 
sono caduti: ateismo, naturalismo, materialismo, fatalismo, indifferentismo, gran parte dei quali, egli precisa, germogliati dall'atomismo. Infine, sostiene l'opportunità - anzi, la necessità morale - dell'intervento censorio della Chiesa Cattolica anche nell'ambito delle scienze naturali. ${ }^{35}$

La prossimità della proposta di Gufl a quella che diventerà, decenni più tardi, la filosofia neo-tomista è notevolissima. Nell'opera del benedettino bavarese è già ben delineata l'architettura concettuale che sosterrà il neotomismo e i paradossi che la caratterizzano. In particolare, è illuminante l'argomentazione di Gufl circa il selectus philosophiae: il systema philosophiae scholasticae è stato a lungo messo alla prova da menti acutissime e non è stato confutato; dunque è talmente improbabile che possa essere confutato che si deve ritenere che non sia possibile confutarlo; dunque non è tollerabile che qualcuno tenti di confutarlo.

La posizione di Gufl deve certamente molto all'evoluzione in senso sistematico della filosofia in ambito tedesco a partire dall'inizio del XVII secolo e, in particolare, a Christian Wolff; non per nulla, il luterano Wolff è uno degli autori ai quali il benedettino bavarese presta maggiore attenzione e verso il quale mostra anche qualche rispetto, pur accusandolo di spinozismo. I pensatori ed eruditi cattolici italiani della seconda metà del Settecento non sembrano aver maturato una nozione di filosofia scolastica altrettanto precisa. Ad esempio, assai minore, tanto rispetto a Brucker che rispetto a Gufl, è la pregnanza che il sostantivo e l'aggettivo scholasticus hanno nell'opera che viene spesso indicata come preludio alla nascita del neo-tomismo: la Summa philosophica ad mentem angelici doctoris s. Thomae Aquinatis, pubblicata dal 1777 al 1783, del domenicano Salvatore Maria Roselli. Il sostantivo scholastici compare nel volume dedicato alla metafisica nella nota 4 a pié di pagina di p. 3-4. In questa nota Rosselli schernisce il paolotto François Jacquier, Antonio Genovesi e Leibniz per aver disprezzato le dottrine, o tentato goffamente di "migliorarle", e le opere metafisiche degli scholastici. Dal testo si evince con chiarezza che, per Roselli, gli scholastici erano stati autori e sostenitori anche di opere e dottrine filosofiche. Nondimeno nell'opera di Roselli, per quanto ho potuto vedere, non si danno occorrenze dell'espressione philosophia scholastica; Roselli parla, piuttosto, di Aristotelici e di philosophia Christiana. ${ }^{36}$

\footnotetext{
${ }^{35}$ GULF, 1750, v. 1, p. (prima serie) 1-104, e v. 2, p. 517-537.

${ }^{36}$ ROSELLUS, 1777-1782, v. 5, Prologus, p. 3.
} 
Interessante è anche il caso dell'uso dell'aggettivo scholasticus nella approbatio di stampa, che compare nel volume dell'opera di Roselli dedicato alla logica, sottoscritta dal carmelitano scalzo Eliseo della Concezione (al secolo Francesco Mango) e datata 2 aprile 1777. Ecco alcuni passaggi di questa lunga approbatio.

Mirari quis forte poterit, quod dum Italorum scriptorum ingenia in libertatem philosophicam se vindicare satagunt, abjectisque servitutis scholasticae vinculis, et auctoritatis, ut ajunt, praejudiciis novam sibi philosophandi viam seligunt, quam eclecticam vocant, idest liberam per singulas sectas sparsarum veritatum electionem, reverendissimus p. magister Salvator Maria Roselli Ordinis Praedicatorum s. Thomae Aquinatis philosophiae nomen dare non dubitet, et ab humo tollendam et sublevandam peripateticam philosophiam non solum manus opitulatrices porrigat, verum etiam pristinum honorem eidem restituere honestissima emulatione contendat. [...] In Logicis Institutionibus tradendis nihil apparet, quod barbaries scholastica dehonestet, atque illae eaedem loquendi formulae, quas tantopere recentiores fastidiunt, squallidum et pannosum habitum deponunt comptiores redditae auctoritatibus priscorum Ecclesiae patrum, et eorumdem loquela consecratae. [...] Opus itaque istud, non solum ad amussim dogmatum catholicae fidei proborumque morum rite exactum praelo dignissimum censeo; verum etiam tamquam exemplar absolutissimum et praestantissimum Christianae philosophiae candidatis omnibus proponere non dubito. ${ }^{37}$

Come il lettore comprende da sé, non siamo di fronte precisamente a un'esaltazione della "scolastica", qualunque cosa Francesco Mango ritenesse che essa fosse. Peraltro, la personalità di questo autore e i suoi interessi suggeriscono che si debba dubitare del fatto che egli ritenesse che la nozione di "scolastica” potesse essere utilizzata per designare qualcosa di indubbiamente positivo. ${ }^{38}$

A partire dal 1761 e fino al 1789 un altro italiano, il monaco celestino Appiano Buonafede, pubblicava i sette volumetti dell'opera Della istoria e della indole di ogni filosofia (stampata in prima edizione dal 1761 al 1781) seguiti dai tre volumetti dell'opera Della restaurazione di ogni filosofia ne' secoli XVI, XVII e XVIII (stampata in prima edizione dal 1785 al 1789)..${ }^{39}$ Quest'opera, già

\footnotetext{
${ }^{37}$ ROSELLUS, $1777-1782$, v. 1, p. 15.

${ }^{38}$ Cf. PAGANO, 1993.

${ }^{39}$ Per comodità di accesso, faccio uso della ristampa ottocentesca dell'insieme delle due opere: 4 voll., Milano, 1837-1838.
} 
oggetto di ottimi studi, ${ }^{40}$ è una sorta di riassunto rielaborato in veste cattolica dell'opera di Brucker. Il lavoro di Buonafede diverge in modo palese da quello di Brucker in pochi aspetti: l'uso della lingua volgare, lo stile gradevolmente letterario, la difesa del cattolicesimo (da intendersi non solo come religione, ma anche come istituzione e come sistema politico-sociale), la difesa di alcuni autori cattolici e la brevità. Per il resto, dipende ampiamente da Brucker e, più in generale, dalla storiografia seicentesca e settecentesca, al punto da condividere con le proprie fonti anche un certo fastidio per la "scolastica". A proposito del gesuita Antonio Rubio, i cui "commentari" alla logica e alla filosofia naturale di Aristotele furono frutto di 22 anni di insegnamento in Messico, Buonafede scrive, in un impasto agrodolce di lode e di biasimo:

Antonio Rubbio [...] fu il primo per cui si ascoltò nell'America la filosofia aristotelica scolastica; e quei buoni indiani, che sentivan ben voglia di altro che di Aristotele, furono stretti ad udirsi dettare da questo ignaziano la logica messicana. ${ }^{41}$

Contrariamente alle aspettative, Buonafede non mette a tema la questione della nozione di "filosofia scolastica". Si può osservare, nondimeno, che l'espressione "filosofia scolastica" compare nei suoi scritti con frequenza ben maggiore dell'espressione "teologia scolastica". È evidente che egli considera l'espressione "filosofia scolastica" legittima e chiara e la utilizza per denotare tutti quegli autori ecclesiastici cattolici che, dal medioevo in avanti, hanno sviluppato il proprio pensiero ricorrendo alla dialettica o alla filosofia aristotelica. Ciò non implica, peraltro, l'identità di filosofia scolastica e di aristotelismo: nel Della restaurazione Buonafede distingue esplicitamente l'aristotelismo coltivato tra tardo medioevo e XVII secolo in aristotelismo genuino, aristotelismo protestante e aristotelismo scolastico; tutti gli scolastici, dunque, sono aristotelici, almeno dal XIII secolo in avanti, ma non tutti gli aristotelici sono scolastici. ${ }^{42}$

\footnotetext{
${ }^{40}$ Ricordo, oltre all'ampio capitolo a opera di Ilario Tolomio nella Storia delle storie generali della filosofia, anche i saggi dedicati dal 1982 al 1998 da Gregorio Piaia a questo scrittore.

${ }^{41}$ BUONAFEDE, 1837-1838, v. 4, p. 56. Buonafede fa qui riferimento alla Logica mexicana, sive Commentarii in universam Aristotelis logicam di Rubio pubblicata in prima edizione nel 1605.

${ }^{42}$ Cf., ad esempio, BUONAFEDE, 1837-1838, v. 4, p. 30-44 (cap. 4 Dei restauratori aristotelici) e p. 45 (ossia una delle pagine iniziali del cap. 5 Degli aristotelici protestanti, p. 44-54). Il cap. 6 dell'opera, poi, s'intitola Degli aristotelici scolastici (p. 54-59). Si può anche osservare che, tra i tre capitoli dedicati agli aristotelici tra tardo medioevo e prima modernità, l'ultimo è anche il più breve.
} 
Ci soffermiamo qui sull'opera di Buonafede anche perché la sua strategia apologetica comprende tre punti particolarmente degni di nota ai fini della presente indagine. Il primo consiste nella distinzione tra una buona scolastica e una cattiva scolastica e in una collocazione degli autori scolastici o entro la prima, o entro la seconda, o in uno stato intermedio tra la prima e la seconda.

[...] distingueremo la moderata scolastica dalla smoderata; e confido che si potrà per questa scorta vedere come la prima fu coltivata con discrete regole non solamente già per lo innanzi da Agostino e da più altri [...] di cui già sopra si è disputato abbastanza, ma da Bernardo, da Lombardo, da Pullo, da Comestore, da Tommaso, da Bonaventura, e da una schiera di altri [...]. Si potrà del pari vedere come la scolastica smoderata temeraria e vuota, che pur troppo esultò sfrenatamente, oltre gli antichi sofisti [...] ebbe maestri e cultori, nei tempi di cui ragioniamo, Berengario e [...] Abelardo, e una gran turba di altri che dieder tanti travagli ai maestri migliori $[\ldots] .^{43}$

Nei cinque capitoli del Della istoria che seguono quello in cui compare il testo ora riportato, ${ }^{44}$ ben pochi autori, in realtà, si salvano dalle reprimende di Buonafede: per la precisione, tutti e soli quelli che, a suo avviso, o posero la ragione (questo, in effetti, è il termine che egli preferisce) e le scienze al servizio della religione e della Chiesa Cattolica, o gettarono i semi della futura scienza sperimentale.

Il secondo punto degno di nota consiste nel proporre una difesa, per così dire, di basso profilo degli "scolastici aristotelici” cinquecenteschi e secenteschi. Parlando di questi autori nell'opera Della restaurazione, Buonafede scrive:

$[\ldots]$ gli scolastici aristotelici nostri [...] se non promossero la restaurazione $<$ della filosofia>, certamente non furono né refrattari, né libertini. Ma siccome il loro numero è grande fuori di misura, così faremo scelta di quelli che furono maggiori in questa, che può dirsi la quarta classe degli scolastici, abbandonando i minori alla polvere e alla dimenticanza. ${ }^{45}$

Buonafede menziona in particolare, pur dedicando al tema solo poche frasi, il valore delle opere giuridiche e controversistiche di alcuni di questi

${ }^{43}$ BUONAFEDE, $1837-1838$, v. 3, p. 373-374. Il passo fa parte del cap. 81 Della filosofia scolastica nelle sue origini dell'opera Della istoria ecc.: p. 367-375.

${ }^{44}$ Tali capitoli sono dedicati ai seguenti temi: cap. 82 Della filosofia scolastica nella sua prima età; cap. 83 Della filosofia scolastica nella seconda età; cap. 84 Della filosofia scolastica nella terza età; cap. 85 Della natura e parti della filosofia scolastica; cap. 86 Di alcune sette scolastiche e di alcune loro particolarità.

${ }^{45}$ BUONAFEDE, 1837-1838, v. 4, p. 55. 
autori. Circa tutti gli altri pensatori appartenenti a questa corrente e ai temi che essi trattarono, il maggior elogio di cui Buonafede li gratifica è il seguente:

[...] se quella scuola fu alquanto irrugginita nell'aristotelismo e nelle quistioni ridondanti e nei parlamenti poco ciceroniani, almeno rimase pura da quei sommi errori da cui furono inondati gli aristotelici genuini, i grecanti, i latinisti, i critici filosofi, di che abbiam disputato dinanzi. Si conceda adunque ingenuamente da noi che gli aristotelici della scuola non seppero restaurare la filosofia, ma non offesero la religione $[\ldots]^{46}$

Il terzo punto degno di nota consiste nell'additare la natura "scolastica" di almeno parte del pensiero sviluppatosi in ambito protestante.

$<$ Brucker> non sa dissimulare che una gran turba de' protestanti suoi amò quanto altri la scolastica aristotelica per gli usi e per gl'interessi della riforma, per le dispute, per le gare e per li desiderj di armeggiare e di vincere; e non sa coprire questa prevaricazione di altro ripiego, salvo che gettando nella oscurità e nella negligenza tutti quei suoi buoni compagni, senza pensare che molti suo malgrado furono illustri, e coloro stessi che con sua licenza fiorirono nella gloria del sincero aristotelismo, quando disputarono sottilmente delle opinioni peripatetiche e con ostinazione settaria le sostennero, se non furono scolastici di nome, lo furono certamente in sostanza. ${ }^{47}$

Le opere ora ricordate di Appiano Buonafede furono ben note agli studiosi cattolici italiani del XIX secolo e l'influenza che esse esercitarono nell'evoluzione della cultura e della politica culturale cattolica ottocentesca meriterebbe ancora qualche ricerca. Non mi è possibile affrontare ora questo tema; constato, tuttavia, che idee molto simili alle tre ora vedute si ritrovano, separatamente, anche in autori della prima metà del XX secolo.

Il passaggio dalla prospettiva degli eruditi cattolici settecenteschi a quella caratteristica della Aeterni Patris fu lento e fulmineo ad un tempo. Fu lento nella misura in cui solo una parte minoritaria degli intellettuali cattolici abbandonò spontaneamente le diverse vie tentate nell'arco di due secoli al fine di conciliare scienze, erudizione e religione cattolica; fu fulmineo nella misura in cui la Aeterni Patris impose per vie politiche agli aderenti alla

\footnotetext{
${ }^{46}$ BUONAFEDE, 1837-1838, v. 4, p. 57.

${ }^{47}$ BUONAFEDE, 1837-1838, v. 4, p. 55. Buonafede fa riferimento al capitolo del quarto volume dell'opera di Brucker intitolato De philosophis genuinam Aristotelis philosophiam sectantibus.
} 
Chiesa Cattolica un modello di filosofia - quello neo-tomista - fino ad allora poco frequentato nello stesso ambito cattolico. ${ }^{48}$ Focalizzando l'attenzione sulla questione qui in esame, ossia la questione relativa al sorgere di un uso consapevole e tematico della nozione di "filosofia scolastica", può essere interessante osservare che l'espressione non compare neppure nella dedica che il domenicano Vincenzo Marreddu prepone all'edizione orvietana del 1859 della Philosophia juxta inconcussa tutissimaque d. Thomae dogmata di Antoine Goudin. ${ }^{49}$ Occorre attendere il 1863 per ritrovare l'espressione nel titolo di un'opera: il Breviarium philosophiae scholasticae del canonico Eugène Grandclaude, pubblicato in prima edizione a Parigi dal 1863 al $1866 .^{50}$ Così commentava La civiltà cattolica l'uscita del primo volume dell'opera.

Nelle scuole cattoliche oramai non si discute più se debbasi o no far ritorno alle dottrine filosofiche di s. Tommaso, ma quale ne sia la più genuina interpretazione, quale il più felice interprete, quale il corso più adatto a farlo intendere e ritenere. Molte di cotali istituzioni in questi ultimi anni sono uscite alla luce in Italia, e qual più qual meno hanno cooperato a far rivivere gl'insegnamenti dell'Angelico Dottore non sol tra noi, ma eziandio di fuori. Godiamo ora di poter annunziare un corso somigliante di filosofia, stampato in Francia. Tre buone qualità vi notiamo: metodo strettamente scolastico, e però attissimo all'insegnamento; ottima distribuzione di materie [...]; e finalmente molta chiarezza d'idee e di stile. Questi tre pregi il raccommandano molto come testo di scuola $\left[\ldots{ }^{51}\right.$

Il commento de La civiltà cattolica è, inutile dirlo, in parte menzognero. Ancora agli inizi degli anni '60 del XIX secolo il "ritorno" a

${ }^{48}$ Gli studi sulle origini e gli sviluppi del neo-tomismo e della neo-scolastica sono numerosi e facilmente reperibili, anche se non molti appaiono rigorosi. Segnalo, invece, alcuni lavori attenti all'evoluzione del contesto politico-culturale entro il quale il neo-tomismo si affermò come l'unica posizione gradita alla Curia romana: MALUSA, 1986-1989; BRAUN, 1989; MALUSA; DE LUCIA, 2001; PAHUD DE MORTANGES, 2005; LEVILLAIN; TICCHI, 2006; GISONDI, 2007; ZAMBARBIERI, 2008.

${ }^{49}$ MARREDDU, 1859.

${ }^{50} \mathrm{Mi}$ riferisco alle opere di ambito teoretico; per quanto riguarda le opere di ambito storico, l'espressione compare, in lingua francese, già nel 1850 nel titolo dell'opera di Jean-Barthélemy Hauréau De la philosophie scolastique. In questo testo, tuttavia, l'espressione aveva un senso ben diverso da quello che avrebbe avuto negli scritti dei neo-tomisti: la scolastica è, per questo autore, innanzi tutto e semplicemente la cultura, dai contenuti più vari, insegnata durante il medioevo nei luoghi dell'istruzione istituzionale; "[...] la philosophie scolastique», scrive in implicita polemica con quanti ne davano una definizione "di contenuto", «est la philosophie professée dans les écoles du moyen-âge» (HAURÉAU, 1850, v. 1, p. 7).

${ }^{51}$ [RECENSORE ANONIMO], 1863. 
Tommaso d'Aquino era una scelta tutt'altro che unanimemente condivisa tra i pensatori cattolici. Il "ritorno" in questione ricevette un impulso decisivo e determinante solamente a seguito dell'azione congiunta di almeno tre fattori. Il primo risiede nel fatto che i predecessori di Leone XIII, ossia Gregorio XVI e Pio IX, avevano già censurato, in molti casi esplicitamente, pressoché tutte le posizioni intellettuali che godessero di qualche fortuna, anche tra la gran parte dei pensatori cattolici, nell'Europa del tempo. Il secondo fattore risiede nella decisa volontà di Leone XIII di portare a compimento la completa sottomissione al papato della Chiesa Cattolica, eliminando ogni residuo in essa di indeterminazione e autodeterminazione. Il terzo fattore risiede nell'adesione non solo dello stesso papa Leone XIII, ma soprattutto del fratello di questi, Giuseppe Pecci, al "tomismo": un insieme di dottrine che, nel corso della prima metà del XIX secolo, alcuni ecclesiastici avevano ritenuto essere state professate da Tommaso d'Aquino. Supportato nella propria decisione dal fratello Giuseppe e circondandosi di personaggi quali Ceferino González, Giovanni Maria Cornodi e Tommaso Maria Zigliara, Leone XIII scelse dunque l'Aquinate come figura al cui pensiero filosofico l'intellettualità cattolica avrebbe dovuto conformarsi.

L'atto formale più celebre di Leone XIII nell'ambito della definizione del tipo di filosofia che i cattolici avrebbero dovuto professare consiste precisamente nell'enciclica Aeterni Patris. A rigore, ciò che l'enciclica stabiliva, ossia una certa concezione della filosofia e l'obbligo di adesione al "tomismo", non era una dogma. Nondimeno, la proclamazione del dogma dell'infallibilità papale, avvenuta appena nove anni prima nel contesto del Concilio Vaticano I, dal punto di vista della Curia rese - e tuttora rende, in verità - di fatto indiscutibile ogni decisione, se non ogni sospiro, papale. Questo fu vero innanzi tutto per la Aeterni Patris e precisamente in questo modo e in questo senso la Curia volle che essa fosse recepita: Roma locuta est; conformarsi a quanto Roma ha detto bisogna. Per di più, la Aeterni Patris fu solamente il primo di una lunga serie di documenti con i quali i successivi tre papi o alcuni organi della Curia romana determinarono quali fossero i contenuti della "sapienza dell'Aquinate", imposero con forza crescente l'obbligo di aderire a essi, e, nel corso del tempo, vincolarono gli intellettuali cattolici specificamente a quelli che vennero individuati come i principi di quella filosofia.

Il lettore potrà trovare l'elenco dei documenti emanati a questo fine tra il 1879 e il 1917 nella nota del card. Pietro Gasparri al canone 1366 del Codex 
iuris canonicis pio-clementino. ${ }^{52}$ Quei documenti segnano anche la continuità tra la condanna delle conquiste civili della modernità contenuta nel Syllabus complectens praecipuos nostrae aetatis errores di Pio IX e la lotta "antimodernista" iniziata dallo stesso Leone XIII e portata alle estreme conseguenze da Pio X. ${ }^{53}$ Ci limitiamo, qui, a ricordare alcuni testi e passi esemplari e a spingerci fino all'inizio degli anni '30 del Novecento. Nell'enciclica Pascendi dominici gregis dell'8 settembre 1907 Pio X così scrive.

Primo igitur ad studia quod attinet, volumus probeque mandamus ut philosophia scholastica studiorum sacrorum fundamentum ponatur. [...] Quod rei caput est, philosophiam scholasticam quum sequendam praescribimus, eam praecipue intelligimus, quae a sancto Thoma Aquinate est tradita: de qua quidquid a Decessore Nostro sancitum est, id omne vigere volumus, et qua sit opus instauramus et confirmamus, stricteque ab universis servari iubemus. Episcoporum erit, sicubi in seminariis neglecta haec fuerint, ea ut in posterum custodiantur urgere atque exigere. Eadem religiosorum ordinum moderatoribus praecipimus. Magistros autem monemus ut rite hoc teneant, Aquinatem deserere, praesertim in re metaphysica, non sine magno detrimento esse. ${ }^{54}$

\section{Nel motu proprio Doctoris Angelici del 19 giugno 1914 Pio X così} scrive.

Doctoris Angelici nemo sincere catholicus eam ausit in dubium vocare sententiam: Ordinare de studio pertinet praecipue ad auctoritatem Apostolicae Sedis qua universalis Ecclesia gubernatur, cui per generale studium, providetur. Quo Nos magno quidem officii munere cum alias functi sumus, tum praesertim die I sept. a. MCMX, quum datis litteris Sacrorum antistitum ad omnes episcopos summosque religiosorum ordinum magistros, quibus cura rite instituendae sacrae iuventutis incumberet, haec in primis eos admonebamus: «Ad studia quod attinet [...]. Eadem religiosorum

52 Codex, 1917, nota a lib. 3, pars 4, tit. 21, can. 1366, \$2, p. 398: «Leo XIII, ep. encycl. Aeterni Patris, 4 aug. 1879; litt. Iampridem, 15 oct. 1879; ep. encycl. Etsi Nos, 15 febr. 1882; ep. Officio sanctissimo, 22 dec. 1887; litt. encycl. Providentissimus Deus, 18 nov. 1893; ep. Inter graves, 1 maii 1804; litt. encycl. Depuis le jour, 8 sept. 1899; Pius X, litt. ap. In praecipuis, 23 ian. 1904; litt. encycl. Pieni l'animo, 28 iul. 1906; ep. Sub exitum, 6 maii 1907; litt. encycl. Pascendi, 8 sept. 1907; motu propr. Sacrorum antistitum, 1 sept. 1910, n. 1; motu propr. Doctoris Angelici, 29 iun. 1914; Benedictus XV, motu propr. Non multo post, 31 dec. 1914; S. C. de Prop. Fide, instr. (ad Vic. Ap. Sin.), 18 oct. 1883, n. IV, 6; S. Studiorum C., 27 iul. 1914; S. C. de Seminariis, 7 mart. 1916».

${ }^{53}$ Sul nesso tra neo-tomismo e anti-modernismo in Pio X, cf. TOLOMIO, 2007. Si veda ovviamente anche VIAN, 2012.

${ }^{54}$ PIUS X, 1907, p. 640. Il testo è ripreso e confermato nel motu proprio Sacrorum antistitum: PIUS X, 1910, p. 656-657. 
ordinum moderatoribus praecipimus». Iam vero, cum dictum hoc loco a Nobis esset "praecipue" Aquinatis sequendam philosophiam, non "unice", nonnulli sibi persuaserunt, Nostrae sese obsequi aut certe non refragari voluntati, si quae unus aliquis e doctoribus scholasticis in philosophia tradidisset, quamvis principiis s. Thomae repugnantia, illa haberent promiscua ad sequendum. At eos multum animus fefellit. Planum est, cum praecipuum nostris scholasticae philosophiae ducem daremus Thomam, Nos de eius principiis maxime hoc intelligi voluisse, quibus, tamquam fundamentis, ipsa nititur. [...] quae in philosophia sancti Thomae sunt capita, non ea haberi debent in opinionum genere, de quibus in utramque partem disputare licet, sed velut fundamenta in quibus omnis naturalium divinarumque rerum scientia consistit [...]. Itaque omnes qui philosophiae et sacrae theologiae tradendae dant operam, illud admonitos iam voluimus, si ullum vestigium, praesertim in metaphysicis, ab Aquinate discederent, non sine magno detrimento fore. - Nunc vero hoc praeterea edicimus, non modo non sequi Thomam, sed longissime a sancto Doctore aberrare eos, qui, quae in ipsius philosophia principia et pronuntiata maiora sunt, illa perverse interpretentur aut prorsus contemnant. Quod si alicuius auctoris vel sancti doctrina a Nobis Nostrisve decessoribus unquam comprobata est singularibus cum laudibus atque ita etiam, ut ad laudes suasio iussioque adderetur eius vulgandae et defendendae, facile intelligitur eatenus comprobata, qua cum principiis Aquinatis cohaereret aut iis haudquaquam repugnaret. ${ }^{55}$

\section{Il 27 luglio del medesimo anno la Sacra Congregazione degli Studi} pubblica il non meno celebre e decisivo documento Theses quaedam, in doctrina sancti Thomae Aquinatis contentae, et a philosophiae magistris propositae, adprobantur. Il documento si presenta come una sorta di approfondimento del precedente motu proprio Doctoris Angelici e fornisce precisamente l'elenco delle tesi che i docenti cattolici dovevano sostenere. ${ }^{56} \mathrm{Si}$ tratta, com'è noto, delle "XXIV tesi del tomismo". Altrettanto noto è che le tesi definite dalla Sacra Congregazione degli Studi sono conformi al tomismo "ortodosso"

\footnotetext{
${ }^{55}$ PIUS X, 1914, p. 336-338.
}

${ }^{56}$ Il testo del decreto della Congregazione così comincia: «Postquam sanctissimus dominus noster Pius papa X motu proprio Doctoris Angelici, edito die XXIX iunii MCMXIV, salubriter praescripsit, ut in omnibus philosophiae scholis principia et maiora Thomae Aquinatis pronuntiata sancte teneantur, nonnulli diversorum Institutorum magistri huic sacrae Studiorum Congregationi theses aliquas proposuerunt examinandas, quas ipsi, tamquam ad praecipua sancti praeceptoris principia in re praesertim metaphysica exactas, tradere et propugnare consueverunt. Sacra haec Congregatio, supra dictis thesibus rite examinatis et sanctissimo Domino subiectis, de eiusdem Sanctitatis Suae mandato, respondet, eas plane continere sancti Doctoris principia et pronuntiata maiora. Sunt autem hae: [...]» (SACRA STUDIORUM CONGREGATIO, 1914, p. 383-384). 
domenicano, ma differiscono da quanto aveva sostenuto Francisco Suárez, e questo - esemplare caso di nemesi storica - creò difficoltà e tensioni tra i gesuiti.

Il codice di diritto canonico pio-benedettino, reso pubblico nel 1917, contiene due canoni lapidari: il 598, $\$ 1$, dedicato alla formazione dei religiosi, e il 1366, $\$ 2$, dedicato alla formazione dei seminaristi.

$\$ 1$ : Religiosi in inferioribus disciplinis rite instructi, in philosophiae studia saltem per biennium et sacrae theologiae saltem per quadriennium, doctrinae d. Thomae inhaerentes ad normam can. 1366, $\$ 2$, diligenter incumbant, secundum instructiones Apostolicae Sedis.

\$2: Philosophiae rationalis ac theologiae studia et alumnorum in his disciplinis institutionem professores omnino pertractent ad Angelici Doctoris rationem, doctrinam et principia, eaque sancte teneant.

Anche Pio XI proseguì, benché con uno stile proprio, la linea politicoculturale dei suoi tre predecessori. Ricollegandosi agli atti di questi ultimi, nell'enciclica Studiorum duce del 29 giugno 1923 così scrive.

Nos vero haec tanta divinissimo ingenio tributa praeconia sic probamus ut non modo Angelicum, sed etiam Communem seu Universalem Ecclesiae Doctorem appellandum putemus Thomam, cuius doctrinam, ut quam plurimis in omni genere litterarum monumentis testata est, suam Ecclesia fecerit. ${ }^{57}$

Nella costituzione apostolica Deus scientiarum Dominus promulgata il 24 maggio 1931, a proposito dei programmi di studio negli studia ecclesiastici stabilisce quanto segue.

In Facultate theologica principem locum teneat sacra theologia. Haec autem disciplina methodo cum positiva tum scholastica tradenda est; ideo veritatibus fidei expositis et ex sacra Scriptura et traditione demonstratis, earum veritatum natura et intima ratio ad principia et doctrinam s. Thomae Aquinatis investigentur et illustrentur. [...] In Facultate Philosophica philosophia scholastica tradatur, eaque ita ut auditores plena cohaerentique synthesi doctrinae ad methodum et principia s. Thomae

${ }^{57}$ PIUS XI, 1923, p. 314. Si noti che il termine scholastica in questo documento non compare in alcuna forma. Va anche detto che l'enciclica di Pio XI ha un tono molto meno aggressivo di quelle di Pio X: in fondo Pio XI, più colto e astuto di Pio X, era più interessato a creare una schiera obbediente di casti devoti che a stigmatizzare teorie filosofiche. 
Aquinatis instituantur. Ex hac autem doctrina diversa philosophorum systemata examinentur et diiudicentur. ${ }^{58}$

Gli effetti in ambito storiografico delle disposizioni papali sono ampiamente noti. Esse hanno fornito un formidabile impulso allo studio del pensiero di Tommaso d'Aquino e, di riflesso, allo studio della filosofia medievale. Come si legge nella Aeterni Patris,

Iamvero inter scholasticos doctores, omnium princeps et magister, longe eminet Thomas Aquinas: qui, uti Caietanus animadvertit, veteres doctores sacros quia summe veneratus est, ideo intellectum omnium quodammodo sortitus est. Illorum doctrinas, velut dispersa cuiusdam corporis membra, in unum Thomas collegit et coagmentavit, miro ordine digessit, et magnis incrementis ita adauxit, ut catholicae Ecclesiae singulare praesidium et decus iure meritoque habeatur. ${ }^{59}$

Se nelle opere di Tommaso d'Aquino è ricapitolato tutto ciò che di vero avevano detto i padri della Chiesa e i pensatori cattolici che l'avevano preceduto, diviene perlomeno legittimo e utile, anche per comprendere lo stesso Tommaso, conoscere il pensiero di quegli autori.

Peraltro, anche i difetti delle indagini storico-filosofiche stimolate dalla Aeterni Patris e dai seguenti documenti ecclesiastici sono ampiamente noti. In quei decreti Tommaso è presentato come un autore dotato di un pensiero coerente e in continuità con quello dei padri della Chiesa; tesi da tempo smentite dalla ricerca. Il pensiero del domenicano medievale, inoltre, veniva - per così dire - scarnificato, distinguendo in esso principi fondamentali e conseguenze accessorie. Di quei pretesi principi venne addirittura stilato un elenco sotto gli auspici del più incolto tra i protagonisti di questa vicenda, ossia Pio X: si tratta, per l'appunto, delle "XXIV tesi del tomismo", le quali ben mostrano quanto poco i tomisti ottocenteschi avessero compreso del pensiero di colui che avevano scelto o accettato come proprio autore di riferimento.

Nel secolo che va dagli anni '70 dell'Ottocento agli anni '80 del Novecento, le imposizioni della Curia romana e la conseguente storiografia neo-tomistica svolsero un ruolo decisivo e tragico. Gli intellettuali cattolici si ritrovarono obbligati a professare quasi per fede non solamente un preciso

\footnotetext{
${ }^{58}$ PIUS XI, 1931, p. 253.

${ }^{59}$ LEO XIII, 1879, p. 408.
} 
insieme di dottrine filosofiche, ma anche una precisa rappresentazione della storia della filosofia e della teologia in genere e della storia della filosofia e della teologia medievali in particolare. Si tatta di un punto importante: da esso dipesero - e tuttora in qualche misura dipendono - non solo gli errori storiografici commessi e difesi da molti storici cattolici della filosofia, ma anche, di riflesso, la concezione che gli storici della filosofia non cattolici ebbero della storia della filosofia medievale. Se, per portare un esempio tra i tanti possibili, Eugenio Garin non fosse stato tratto in inganno dalla erudizione di Étienne Gilson e non si fosse fidato della falsa rappresentazione della storia della filosofia medievale che questi andava proponendo, probabilmente avrebbe sviluppato una concezione dei rapporti tra filosofia medievale, filosofia rinascimentale e filosofia moderna diversa da quella che egli propugnò.

Tra le tesi storiografiche che gli storici cattolici della filosofia si trovarono a dover sostenere vi fu quella della "decadenza" del pensiero posteriormente all'opera di Tommaso d'Aquino. Questa tesi è contenuta già nella Aeterni Patris. A rigori, si potrebbe osservare che in questa enciclica si legge anche quanto segue.

Illud etiam accedit, quod philosophicas conclusiones Angelicus Doctor speculatus est in rerum rationibus et principiis, quae quam latissime patent, et infinitarum fere veritatum semina suo velut gremio concludunt, a posterioribus magistris opportuno tempore et uberrimo cum fructu aperienda. $^{60}$

Tuttavia, la ricezione di Tommaso d'Aquino che l'enciclica impone sul piano teoretico e afferma esservi stata sul piano storico è meramente passiva o strumentale; il giudizio che la Aeterni Patris dà della storia della filosofia e della cultura in genere posteriori all'Aquinate, anche in ambito ecclesiastico, è nitidamente negativo.

[...] iudicamus temere esse commissum, ut eidem suus honos non semper, nec ubique permanserit: praesertim cum philosophiae scholasticae et usum diuturnum et maximorum virorum iudicium, et, quod caput est, Ecclesiae suffragium favisse constaret. Atque in veteris doctrinae locum nova quaedam philosophiae ratio hac illae successit, unde non ii percepti sunt fructus optabiles ac salutares, quos Ecclesia et ipsa civilis societas maluissent. Adnitentibus enim novatoribus saeculi XVI, placuit philosophari citra quempiam ad fidem respectum, petita dataque vicissim

${ }^{60}$ LEO XIII, 1879, p. 408-409. 
potestate quaelibet pro libitu ingenioque excogitandi. Qua ex re pronum fuit, genera philosophiae plus aequo multiplicari, sententiasque diversas atque inter se pugnantes oriri etiam de iis rebus, quae sunt in humanis cognitionibus praecipuae. A multitudine sententiarum ad haesitationes dubitationesque persaepe ventum est: a dubitationibus vero in errorem quam facile mentes hominum delabantur, nemo est qui non videat. - Hoc autem novitatis studium, cum homines imitatione trahantur, catholicorum quoque philosophorum animos visum est alicubi pervasisse; qui patrimonio antiquae sapientiae posthabito, nova moliri, quam vetera novis augere et perficere maluerunt, certe minus sapienti consilio, et non sine scientiarum detrimento. ${ }^{61}$

Étienne Gilson aveva certamente presenti queste parole quando, assumendo e adattando ai propri scopi idee di matrice idealistica e cousiniana, sostenne in alcune conferenze il darsi nella storia della filosofia di un processo ricorrente, espressione di leggi interne al pensiero stesso. Il processo teorizzato da Gilson si articola in quattro fasi: formulazione del sistema, crisi del sistema, scetticismo, moralismo/misticismo. Inutile dire che, secondo Gilson, Tommaso d'Aquino rappresenta il momento del "sistema" e tutto ciò che lo segue fino al XVIII secolo - innanzi tutto Guglielmo di Ockham - rappresenta il momento della corrosione del sistema, dello scetticismo e, infine, della reazione, nelle forme del moralismo e del misticismo, allo scetticimo stesso. ${ }^{62}$ Era il 1937; quattro anni dopo, nel 1941, il gesuita Carlo Giacon pubblicava la monografia Guglielmo di Occam. Saggio storico-critico sulla formazione e sulla decadenza della Scolastica.

Il titolo dell'opera ben esprime le convinzioni storiografiche del suo autore al tempo in cui insegnava "esposizione della dottrina e della morale cattolica” presso l'Università Cattolica di Milano. Scoto - sostiene Giacon critica, fraintendendolo, Tommaso d'Aquino; Ockham, a sua volta, critica, in questo caso giustamente, Scoto. Così facendo, però, Ockham si allontana ulteriormente da Tommaso e fonda, remotamente, la filosofia moderna. È dunque opportuno conoscere il pensiero di Ockham, scrive Giacon, al fine di avere «[...] una spiegazione e una giustificazione dell'abbandono e del ripudio della filosofia scolastica da parte dei filosofi dell'Umanesimo, del Rinascimento e da Cartesio in poi $[\ldots] » .{ }^{63}$

\footnotetext{
${ }^{61}$ LEO XIII, 1879, p. 411.

${ }^{62}$ GILSON, 1937.

${ }^{63}$ GIACON, 1941, p. ix-x, citazione da p. x.
} 
Si tratta di tesi storiografiche ampiamente diffuse, in quegli anni, in ambito cattolico e su di esse, in questa sede, non occorre dire altro. Ciononostante, questo volitivo gesuita aveva in serbo un colpo d'ala. Se ne scorge un primo cenno già nella conclusione del saggio su Ockham.

Dopo la prima decadenza della Scolastica, avvenuta nei secoli XIV e XV, vi fu un rifiorire di essa nei secoli XVI e XVII, sia per la necessità intrinseca della rivalutazione di una speculazione ricchissima, dinanzi alla povertà delle nuove ricerche filosofiche e teologiche, sia per la necessità della polemica contro il Protestantesimo. Un merito particolare in questa prima rinascita della Scolastica ebbero i grandi teologi domenicani commentatori di s. Tommaso: il card. Gaetano, Silvestro da Ferrara, il Vittoria, il Soto, il Cano, il Báńez; un merito non meno importante ebbero i teologi della nascente Compagnia di Gesù: il Toleto, il Fonseca, il Molina, il Vásquez, il Lessio, Gregorio di Valenza, e s. Roberto Bellarmino. Un posto del tutto singolare lo rivendica a sé Francesco Suárez. Egli non volle essere un semplice commentatore di dottrine altrui. Volle impadronirsi della speculazione scolastica per esporla in modo proprio e secondo le necessità dei nuovi tempi. ${ }^{64}$

Come si vede, nel 1941 Giacon aveva già formulato l'idea secondo la quale vi era stata una "scolastica dei tempi d'oro", alla quale era seguito un periodo di decadenza, il quale era stato a sua volta seguito da una "rifioritura della scolastica". Si noti anche che, in questo momento della sua evoluzione intellettuale, Giacon presenta gli autori che ha menzionato qualificandoli come teologi. Non nega per nulla che nelle loro opere siano presenti anche dottrine filosofiche, tuttavia quella qualifica non è, nell'economia del discorso di Giacon, priva di ragione. Questa ragione emerge in uno scritto di Giacon di poco posteriore: il saggio pubblicato nel $1942 \mathrm{con}$ il titolo Galileo e la scolastica della decadenza. Conferenza tenuta sotto gli auspici della Reale accademia d'Italia all'Università cattolica del S. Cuore il giorno 12 maggio 1942 - XX.

Le tesi formulate in questo testo da Giacon presentano una certa complessità e mostrano lo sforzo di articolare lo schema "età dell'oro - decadenza - rifioritura" a fronte delle sfaccettature del quadro storico tardo-medievale e rinascimentale e, soprattutto, dello scandalo costituito dal "caso Galilei". Ecco, in sintesi, la tesi di Giacon. La decadenza che caratterizzò la "scolastica della decadenza” riguardò non solamente la metafisica, ma anche, per così dire, l'epistemologia; specificamente, fu perduta non solo la comprensione

${ }^{64}$ GIACON, 1941, p. 679. 
dei primi principi metafisici, ma anche la distinzione tra fisica filosofica e scienza fisica. ${ }^{65} \mathrm{Nel}$ corso del Cinquecento si diede sì una rifioritura della filosofia scolastica, tuttavia gli "uomini eminenti" che ne furono protagonisti ebbero interessi principalmente teologici; la rinascita, dunque, riguardò non la filosofia tout court, bensì la filosofia concepita come propedeutica alla teologia ${ }^{66}$ Gli aristotelici rinascimentali che si occuparono di fisica non furono autori scolastici; furono, invece, pensatori - in parte averroisti, in parte alessandristi - che avevano smarrito la comprensione dell'autentico pensiero aristotelico sia in ambito metafisico che in ambito fisico. ${ }^{67}$ Galilei né fu istruito nell'autentica metafisica aristotelica, né si occupò di essa, né polemizzò con la corrente di filosofia scolastica che si interessava di questioni teologiche; i suoi nemici furono piuttosto gli aristotelici non scolastici. ${ }^{68}$ Galilei fu nel giusto nel criticare la fisica professata da quegli aristotelici e non fu compreso, a causa della loro impreparazione, dagli scolastici teologi. ${ }^{69}$

Non vi fu, sventuratamente, accanto al genio di Galileo, un genio veramente filosofico, aperto alle più larghe concezioni metafisiche, capace di illustrare con i principi eterni della ragione le nuove speculazioni scientifiche, fino a inquadrarle in una nuova visione generale della realtà fisica. Molti fisici di nessun valore, e alcuni teologi ignari di scienze, di genuina metafisica e di esegesi biblica, ebbero l'impressione che le innovazioni galileiane facessero rovinare tutto il sapere umano, e gli furono ostinati avversari. La generazione dei pedanti scolastici della decadenza, che non erano, come s’è visto, né teologi difensori della verità religiosa, né filosofi seguaci della metafisica aristotelico-tomistica, ma scienziati degeneri, dimentichi dei canoni scientifici lasciati dai grandi pensatori greci e scolastici, scomparve sotto i colpi della polemica galileiana. ${ }^{70}$

Giacon chiuse la propria conferenza del 1942 citando un'affermazione di Giovanni Gentile, secondo il quale «Gli aristotelici avversari di Galilei appartenevano al periodo della decadenza». ${ }^{71} \mathrm{La}$ collocazione e la periodizzazione di "altezze" e "abissi" proposte da Giacon erano, in realtà,

\footnotetext{
${ }^{65}$ GIACON, 1942, p. 17-25.

${ }^{66}$ GIACON, 1942, p. 6-7.

${ }^{67}$ GIACON, 1942, p. 8.

${ }^{68}$ GIACON, 1942, p. 11-13, 16-17 e 47-49.

${ }^{69}$ GIACON, 1942, p. 50-52.

${ }^{70}$ GIACON, 1942, p. 50.

${ }^{71}$ GIACON, 1942, p. 52.
} 
ben diverse da quelle evocate da Gentile, tuttavia nell'anno ventesimo dell'era fascista, in perdurante luna di miele tra regime fascista e cattolicesimo papalino, su certe sottigliezze si poteva sorvolare.

La piena applicazione al campo della filosofia della tesi circa la "rinascita" della scolastica ha luogo nella guida bibliografica pubblicata da Giacon nel 1943 con il titolo Il pensiero cristiano con particolare riguardo alla scolastica medievale. In esso compare per la prima volta l'espressione "seconda scolastica", dando così finalmente un nome al "rifiorimento" della scolastica di cui Giacon aveva già parlato nei precedenti scritti. Il gesuita padovano così presenta, nella sintetica prefazione che apre il volume, la propria proposta storiografica.

[...] la presente Guida riguarda la storia della filosofia cristiana dalla patristica alla neoscolastica; siccome però soprattutto nel medio evo si ebbe la costituzione e l'elaborazione di una filosofia cristiana in senso rigoroso, mentre prima si trattò soltanto di una preparazione remota, e dopo di vicende, di echi e di sviluppi, così la parte maggiore della Guida è stata riservata alla filosofia cristiana medievale. La speculazione di questa parte di storia della filosofia è comunemente chiamata con l'appellativo di scolastica. Il periodo di svolgimento del pensiero cristiano scolastico va sostanzialmente dal secolo IX al secolo XV, cioè dalla rinascita carolingia alla fine del medio evo, dai primi dialettici alla fine del nominalismo. A questo primo periodo, che termina con la decadenza della scolastica, segue un altro periodo, quello del rifiorimento della medesima, chiamato talvolta col nome di scolastica spagnola, prima e dopo il Concilio di Trento, fino a tutto il secolo XVIII, quando si verifica una sua nuova decadenza. In fine, nei secoli XIX e XX si ha un secondo rifiorimento: la neoscolastica e soprattutto il neotomismo. Per comodità di espressione, ricordando le tre accademie platoniche, si è convenuto di chiamare prima, seconda e terza scolastica i tre periodi della filosofia cristiana: medievale, spagnolo e neoscolastico. Essi, insieme a un'introduzione generale e alla patristica, costituiscono pertanto le cinque sezioni della presente Guida. ${ }^{72}$

La tesi storiografica di Giacon giunge infine a maturazione nei tre volumi dell'opera La seconda scolastica. Pubblicati dal 1944 al 1950, essi diedero corpo a quella proposta e ne sancirono il successo. Nelle prefazioni ai tre volumi, ${ }^{73}$ Giacon dichiara ripetutamente ed esplicitamente il fine che lo ha guidato nella propria ricerca: contribuire alla ricostruzione nazionale

\footnotetext{
${ }^{72}$ GIACON, 1943, p. xiii-xiv.

${ }^{73}$ GIACON, $1944-1950$, v. 1, p. 5-12, v. 2, p. 1-9 e v. 3, p. 5-9.
} 
italiana propugnando la validità di un sistema filosofico, quello neo-tomistico, solido e capace di dare fondamento tanto alle scienze sperimentali, quanto a ciò che vi è di vero - sebbene solamente in forma di frammenti - nel pensiero contemporaneo. Parimenti, Giacon dichiara esplicitamente di non aver inteso scrivere un'opera erudita: ha inteso invece, precisa in più punti, contribuire al progresso del pensiero contemporaneo; per questa ragione, prosegue, ha scelto di trattare solamente quegli autori e quei temi che, a suo avviso, erano utili al suo scopo, lasciando il resto nell'oblio.

In modo non meno esplicito Giacon enuncia, fin dalla prima pagina del primo volume dell'opera, la propria visione della storia e della storia della filosofia dei secoli dal XIV al XVIII.

È noto che, dopo l'età classica [ossia il XIII secolo], con l'avvento e il trionfo del nominalismo, mentre prendevano vigore correnti di pensiero antiscolastiche, in tempi turbolenti e poco propizi alla speculazione, per la mancanza di menti elette, che potessero reggere al confronto con i grandi maestri del secolo XIII, la filosofia scolastica era entrata in un periodo di profonda decadenza. In particolare la cattività avignonese, le lotte tra papato e impero, lo scisma d'Occidente, i Concili di Costanza e di Basilea, i movimenti ereticali di Wicliff e di Huss avevano messo e mettevano la suprema autorità ecclesiastica nell'impossibilità di promuovere efficacemente gli studi, come aveva fatto nei secoli precedenti. [...] Gli ordini religiosi [...], rilassandosi alquanto nel rigore della disciplina, non erano più i cenacoli della più pura attività di pensiero. Rassodata però, nella seconda metà del '400, la Sede romana, [...] fu possibile una rinascita di quella filosofia che $[\ldots]$ aveva reso splendido il miglior secolo del medio evo. ${ }^{74}$

Un testo siffatto non può non suscitare, in un lettore moderno, fastidio o riso. Se, però, si prosegue nella lettura, si percepisce il lavorio intellettuale di Giacon, quasi costretto dal proprio oggetto a non ignorare le reali sembianze degli autori di cui si andava occupando. Scopriamo, dunque, che Giacon nega che ciò che è seguito a Tommaso d'Aquino sia stato sempre e solo decadenza o semplice passiva ripetizione del pensiero dell'Aquinate; ammette un qualche contributo dell'umanesimo a quella che egli ritiene essere la rinascita cinquecentesca della scolastica; riconosce la presenza, nei secoli di cui si occupa, anche di tradizioni universitarie non tomiste, salvo attribuire loro il solo compito di pungolare il tomismo; vede e segnala la natura composita del pensiero degli autori che egli tratta, salvo giudicarla negativamente;

${ }^{74}$ GIACON, 1944-1950, v. 1, p. 5. 
non risparmia una stoccata al domenicano “ortodosso" Domingo Báńez, accostandolo a Cornelius Jansen.

In tutte e tre le introduzioni ai volumi di La seconda scolastica Giacon presenta il piano completo dell'opera. Così egli scrive nell'introduzione al secondo volume.

[...] al periodo del secolo XVI precedente il Concilio di Trento, davo come titolo proprio: I grandi Commentatori di s. Tommaso: il Gaetano, il Ferrarese, il Vitoria; e avrei dato al periodo posteriore al medesimo Concilio, il titolo: I nuovi problemi etico-giuridici; al secolo XVII: Le sistemazioni generali; al secolo XVIII: La nuova decadenza. ${ }^{75}$

I tre volumi pubblicati da Giacon comprendono solo i primi due dei quattro temi, o titoli, ora ricordati; l'opera è dunque, in realtà, incompiuta. Non ho svolto ricerche per stabilire le ragioni che spinsero Giacon a non portare a termine il progetto iniziale. Mi piacerebbe pensare che così accadde perché, leggendo testi e raccogliendo materiali, lo stesso Giacon si rese conto del fatto che l'"autentico spirito del tomismo" era una chimera che gli stessi autori che egli riteneva "scolastici" potevano contribuire a dissipare; è questa, però, solamente una nuda ipotesi. Si può invece dire con ragionevole certezza che la tesi storiografica di Giacon costituisce un'articolazione del paradigma storiografico neo-tomista. Grazie a questa tesi, la tradizione dottrinale che si ispira all'Aquinate non è più trascinata nel gorgo della filosofia moderna innescato da Guglielmo di Ockham; al contrario, essa, dopo un periodo di latenza, si ripresenta alla ribalta della storia, seppure in forme imperfette, e conserva le aspirazioni e i materiali che renderanno possibile quella che, secondo Giacon, è la più recente e autentica rifioritura della scolastica medievale, ossia la neo-scolastica e il neo-tomismo.

Precisamente a causa del fatto che questo schema non rispetta l'originale dettato storiografico neo-tomista, non tutti i cultori di quel dettato sono stati disposti ad accogliere la tesi di Giacon: se si ammette che non tutto ciò che segue Tommaso è traviamento del suo pensiero, diviene difficile tracciare confini nitidi tra la vera e la falsa filosofia. Un autore ostile, almeno di fatto, alla proposta storiografica di Giacon fu lo stimmatino Cornelio Fabro. Fabro riteneva che l'"autentico spirito del tomismo" non fosse stato compreso da nessuno dei seguaci dell'Aquinate. Il suo giudizio negativo nei confronti di

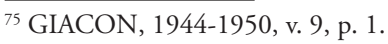


Francisco Suárez risaliva perlomeno all'articolo Neotomismo e neosuarezismo: una battaglia di principi, pubblicato nel 1941. Neppure gli autori appartenenti all'Ordine dei Frati Predicatori, tuttavia, riscossero la sua approvazione. Nel 1958 Fabro pubblicò l'articolo L'obscurcissement de l'esse" dans l'école thomiste e ancora nel 1989 poneva in esergo a un proprio articolo dedicato a João Poinsot (noto anche come Ioannes a s. Thoma) il seguente aforisma: «Succede spesso, nel seguire la via dei Commentatori, come a quel viaggiatore diretto a Londra: 'Conduce questa strada a Londra? - Certo. Ma se volete giungervi, bisogna che vi voltiate"». Di fronte alla domanda "Che cosa hanno detto, fatto, insegnato... i tomisti - rispetto all'insegnamento del maestro s. Tommaso in questi sette secoli» e alla constatazione che «[...] i discepoli dell'Angelico fin da principio e in modi diversi si sono divisi fra loro", Fabro si chiede, non retoricamente: «Dobbiamo allora dire cioè ammettere che la cosiddetta scuola tomistica, fin dal suo inizio, mostra una tendenza deviazionistica in atto dalla rivoluzione speculativa riconosciuta al Maestro?». La risposta di Fabro è positiva: la scuola tomistica tradì fin dai suoi esordi i capisaldi del pensiero dell'Aquinate. $^{76}$

Nonostante che idee quali quelle di Fabro abbiano goduto di ampio credito in ambito cattolico, la visione storiografica di Giacon ha comunque avuto vastissima risonanza e seguito. In effetti, essa ha permesso di dare una collocazione ad autori altrimenti esclusi da ogni narrazione storica o malamente rinchiusi entro l'inadeguata categoria di "scolastica spagnola". Questa soluzione è risultata gradita agli storici della filosofia di ogni orientamento: sia a quelli impigliati nei lacci delle disposizioni pontificie, sia a quelli abbacinati da decenni - per non dire da secoli - di polemiche anticlericali. Ai primi permetteva di vantare una qualche sopravvivenza della "vera filosofia" anche nei secoli della Riforma e della prima modernità; ai secondi permetteva di circoscrivere e rimuovere con maggior agio, quasi con un unico gesto della mente e della penna, tutti coloro che, a loro avviso, si erano opposti alla rinascita e allo sviluppo dell'autentica "vera filosofia". Forse non è del tutto inutile segnalare che tanto la rappresentazione generale della storia della filosofia sostenuta da Giacon quanto quella simmetricamente propugnata da molti autori "laici" del XX secolo si fondavano sulla subordinazione dell'indagine storica a un programma teoretico e, per ciò che di storico contenevano, sono state significativamente smentite dalla ricerca; le nozioni di "scolastica" e

${ }^{76}$ FABRO, 1989, p. 56-58. 
"seconda scolastica" potrebbero dunque essere oggi felicemente abbandonate, ${ }^{77}$ o dovrebbero perlomeno essere soggette a un profondo ripensamento. ${ }^{78}$

Ciononostante, l'idea che vi siano stati un apogeo, un declino e una "risorgenza" di una filosofia detta "scolastica", o addirittura "tomistica", è tutt'ora ampiamente diffusa, specialmente in ambito cattolico, ${ }^{79}$ e molti studiosi, soprattutto non italiani, non sembrano consapevoli delle prospettive ideologiche e storiografiche che questa tesi e questa terminologia veicolano. Invero, si può rinvenire questa idea anche in vesti inattese. In un'intervista rilasciata il 19 agosto 2013 a L'osservatore romano, Jorge Mario Bergoglio, da pochi mesi papa con il nome di Francesco, così diceva.

L'uomo è alla ricerca di se stesso, e ovviamente in questa ricerca può anche commettere errori. La Chiesa ha vissuto tempi di genialità, come ad esempio quello del tomismo. Ma vive anche tempi di decadenza del pensiero. Ad esempio: non dobbiamo confondere la genialità del tomismo con il tomismo decadente. Io, purtroppo, ho studiato la filosofia con manuali di tomismo decadente..$^{80}$

Queste parole hanno un che di sorprendente. Prendendo il discorso così come esso suona, parrebbe trattarsi di un attacco al neo-tomismo e di una rivalutazione dei seguaci più antichi dell'Aquinate. Quella che, nelle intenzioni e negli intransigenti atti di quattro papi, avrebbe dovuto essere la vera rifioritura - anzi, l'inveramento - del tomismo, parrebbe ora divenuta, nelle parole dell'attuale papa, un momento di declino. Probabilmente non ho ben compreso il pensiero di Bergoglio; se, però, il lettore mi permette una battuta, dirò che tutto, a questo mondo, è il declino di qualcosa.

77 È ciò che mi sono permesso di sostenere in FORLIVESI, 2013.

${ }^{78}$ A titolo di esempio, osservo che BRETT, 2000, ha proposto la seguente reinterpretazione della nozione di "seconda scolastica": vi è una "seconda" scolastica allorché, e nella misura in cui, la scolastica tenta di ridefinire il proprio approccio alla Sacra Scrittura a fronte delle metodologie esegetiche degli umanisti e dei protestanti.

${ }^{79}$ GRACIA, 1998, non solamente ha riproposto e difeso la nozione di "età d'argento della scolastica", ma ha reintrodotto in essa la curvatura ispanocentrica che già Giacon aveva superata.

${ }^{80}$ SPADARO, 2013, p. 8. 
FORLIVESI, Marco. The historiographical problem of the notion of "scholastic philosophy" and the genesis of the notion of "second scholasticism". Trans/Form/Ação, Marília, v. 37, p. 169-208, 2014. Edição Especial.

\begin{abstract}
The article aims to elucidate the genesis of the concepts of "scholastic philosophy" and "second scholasticism". Past researches on the history of the concept of "scholasticism" do not seem to have addressed the possible difference between the history of the concept of "scholastic philosophy" and the history of the concepts of "scholastic theology" and "scholasticism". In this study, the thesis is advanced that the history of the concept of "scholastic philosophy" is interrelated but not identical with the history of those two concepts, and clues are presented about the timing and manner in which, from early modern times to the beginning of the twentieth century, the adjective "scholasticus" was used to characterise a type of philosophy which is conceived to be at variance with that practiced by modern authors. The results of this inquiry provide the basis for determining the reasons that, in the 1940 s, led Carlo Giacon to formulate the notion of "second scholasticism", a notion which displays Giacon's historiographical convictions and theoretical perspectives.
\end{abstract}

KEYWORDS: Scholastic philosophy. Second scholasticism. Neo-scholasticism. Carlo Giacon. History of philosophical historiography.

\title{
Bibliografia
}

BAYLE, Pierre. Dictionaire historique et critique. Amsterdam: Chez P. Brunel, 1740.

BRAUN, Karl-Heinz (Hrsg.). Kirche und Aufklärung - Ignaz Heinrich von Vessenberg (1774-1860). München-Zürich: Verlag Schell \& Steiner, 1989.

BRETT, Annabel S.. Authority, reason and the self-definition of theologians in the spanish "Second Scholastic". In: TUCKER, George Hugo (ed.). Forms of the "Medieval" in the "Renaissance": a multidisciplinary exploration of a cultural continuum. Charlottesville: Rookwood Press, 2000. p. 63-89.

BRUCKERUS, Iacobus. Historia critica philosophiae. Lipsiae: Apud Bernh. Christoph. Breitkopf, 1743. v. 3.

BUONAFEDE, Appiano. Della istoria e della indole di ogni filosofia e della ristaurazione di ogni filosofia nei secoli XVI, XVII, XVIII. Milano: Dalla Società Tipografica de' Classici Italiani, 1837-1838. 4v. Il titolo dell'opera presenta varianti grafiche nei frontespizi primari e secondari dei diversi volumi.

CODEX iuris canonicis Pii X pontificis maximi iussu digestus, Benedicti papae XV auctoritate promulgatus. Prefatione, fontium annotatione et indice analytico-alphabetico ab e. Petro card. Gasparri auctus. Romae: Typis poliglottis Vaticanis, 1917. 
CREMONINO, Cesare. Le nubi. MONTANARI, Umberto. L'opera letteraria di Cesare Cremonini. Cesare Cremonini (1550-1631). Il suo pensiero e il suo tempo. Cento: Centro studi Girolamo Baruffaldi, 1990. p. 151-226.

DE BENEDICTIS, Giovanni Battista. Lettere apologetiche in difesa della teologia scolastica e della filosofia peripatetica. Napoli: Nella stamperia di Giacomo Raillard, 1694.

DE HANGEST, Jérôme. A difesa dell'Università. A cura di Riccardo Quinto. Padova: CLEUP, 2009.

DONATO, Maria Pia. Scienza e teologia nelle congregazioni romane. La questione atomista, 1626-1727. In: ROMANO, Antonella (éd.). Rome et la science moderne: entre Renaissance et Lumières. Rome: Publications de l'École Française de Rome, 2009. p. 595634.

DUPASQUIER, Sebastianus. Summa philosophiae scholasticae et Scotisticae. Lugduni: Sumptibus Antonii Briasson, 1692. 4v.

FABRO, Cornelio. Neotomismo e neosuarezismo: una battaglia di principi. Divus Thomas (P.), v. 44, p. 168-215 e 420-498, 1941. 472, 1958 .

L'obscurcissement de l'“esse” dans l'école thomiste. Revue Thomiste, v. 3, p. 443-

. Il posto di Giovanni di s. Tommaso nella scuola tomistica. Angelicum, v. 66, p. 56-90, 1989.

FORLIVESI, Marco. La filosofia universitaria tra XV e XVII secolo. Padova: CLEUP, 2013.

GIACON, Carlo. Guglielmo di Occam: saggio storico-critico sulla formazione e sulla decadenza della Scolastica. Milano: Vita e Pensiero, 1941. L'opera è divisa in due tomi ma con continuità nella numerazione delle pagine.

- Galileo e la scolastica della decadenza: conferenza tenuta sotto gli auspici della Reale accademia d'Italia all'Università cattolica del S. Cuore il giorno 12 maggio 1942 - XX. Gallarate: Istituto filosofico Aloisianum 1942.

. Il pensiero cristiano con particolare riguardo alla scolastica medievale. Milano: Vita e Pensiero, 1943.

. La seconda scolastica. Milano: Fratelli Bocca, 1944-1950. 3v.

GILSON, Étienne. The Unity of philosophical experience. New York: Charles Scribner's Sons, 1937.

GISONDI, Antonio. "Recta ratio", ragione naturale, filosofia perenne tra Settecento e Ottocento. In: DI CERBO, Valentino (a cura di). Alfonso Maria Iannucci e la teologia neoscolastica. Bologna: EDB, 2007. p. 55-114.

GRACIA, Jorge. Suárez (and later scholasticism). In: MARENBON, John (ed.). Medieval philosophy. London: Routledge, 1998. p. 452-474. (Routledge History of Philosophy, v. 3) 
GUFL, Veremundus. Philosophia scholastica universa principiis s. Thomae d. a. apprime conformata, et contra neotericos praecipue defensa, experimentorum quoque et matheseos tum purae tum mixtae accessionibus aucta. Ratisbonae: Typis et sumptibus Christiani Theophili Seiffarti, 1750. 4v.

HAURÉAU, Jean-Barthélemy. De la philosophie scolastique. Paris: Pagnerre: 1850. 2v.

LEO XIII. Epistola encyclica Aeterni Patris. In: ACTA Sanctae Sedis. Romae: Typis polyglottae officinae S. C. De Propaganda Fide, 1879. v. 12, p. 97-115.

LEVILLAIN, Philippe; TICCHI, Jean-Marc (éds.). Le pontificat de Léon XIII: renaissances du Saint-Siège? Roma: École Française de Rome, 2006.

LINCAEUS, Richardus. Universa theologia scholastica. Salmanticae: Ex officina Melchioris Estevez, 1679.

LLAMAZARES, Thomas. Cursus philosophicus, sive Philosophia scholastica, ad mentem Scoti, nova et congruentiori addiscentibus methodo disposita. Lugduni: Sumptibus Ioan. Anton. Huguetan, et Guillielmi Barbier, 1670.

LYNCEUS, Richardus. Universa philosophia scholastica. Lugduni: Sumptibus Philippi Borde, Laurentii Arnaud, et Claudii Rigaud, 1654. 3v.

MABILLON, Jean. Traité des études monastiques. Paris: Chez Charles Robustel, 1691.

MALUSA, Luciano. Neotomismo e intransigentismo cattolico. Milano: IPL, 1986-1989. 2v.

.; DE LUCIA, Paolo (a cura di). Chiesa e pensiero cristiano nell'Ottocento: un dialogo difficile. Genova: Brigati 2001.

MARREDDU, Vincentius. Rev. in Christo patri fr. Alexandro Vincentio Jandel Ordinis Praedicatorum generali magistro felicitatem. In: GOUDIN, Antonius. Philosophia juxta inconcussa tutissimaque d. Thomae dogmata. Urbeveteri: Prelis Speraindeo Pompei, 1859. p. $\mathrm{v}-\mathrm{xv}$.

MORHOF, Daniel Georg. Polyhistor sive de notitia auctorum et rerum commentarii. Lubecae: Sumptibus Petri Böckmanni, 1688.

PAGANO, Antonella. Eliseo della Concezione. In: DIZIONARIO Biografico degli Italiani. Roma: Istituto della Enciclopedia Italiana, 1993. v. 42. Disponibile < www. treccani.it/ enciclopedia/ eliseo-della-concezione_(Dizionario-Biografico)/ > Consultato il 29/09/2014.

PAHUD DE MORTANGES, Elke. Philosophie und kirchliche Autorität. Der Fall Jakob Frohshammer vor der römischen Indexkongregation (1855-1864). Paderborn: Ferdinand Schöningh, 2005.

PIUS X. Epistola encyclica Pascendi dominici gregis. In: ACTA Sanctae Sedis. Romae: Ex Typographia polyglotta S. C. de Propaganda Fide, 1907. v. 40, p. 593-650.

Motu proprio Doctoris Angelici. In: ACTA Apostolicae Sedis. Romae: Typis poliglottis Vaticanis, 1914. v. 6, p. 336-341. 
Motu proprio Sacrorum antistitum. In: ACTA Apostolicae Sedis. Romae: Typis poliglottis Vaticanis, 1910. v. 2, p. 655-680.

. Littera encyclica Studiorum duce. In: ACTA Apostolicae Sedis. Romae: Typis poliglottis Vaticanis, 1923. v. 15, p. 309-326.

. Constitutio apostolica Deus scientiarum Dominus. In: ACTA Apostolicae Sedis. Romae: Typis poliglottis Vaticanis, 1931. v. 23, p. 241-262.

POPPI, Antonino. La scientificità del discorso teologico nella Scuola padovana del Seicento. In: PIAIA, Gregorio (a cura di). La presenza dell'aristotelismo padovano nella filosofia della prima modernità. Roma-Padova: Antenore, 2002. p. 339-370. Il saggio è pubblicato anche in POPPI, Antonino. Ricerche sulla teologia e la scienza nella Scuola padovana del Cinque e Seicento. Soveria Mannelli: Rubbettino Editore, 2001. p. 101-123.

QUINTO, Riccardo. "Scholastica”: storia di un concetto. Padova: Il Poligrafo, 2001.

[RECENSORE ANONIMO], [Recensione di GRANDCLAUDE, Eugène. Breviarium philosophiae scholasticae. Parisiis 1863. v. 1]. La civiltà cattolica, v. 14, p. 348, 1863.

ROSELLUS, Salvator Maria. Summa philosophica ad mentem angelici doctoris s. Thomae Aquinatis.) Romae: Ex typographia fratrum Puccinelli, 1777-1783. 6v. (il v. 1 fu pubblicato nel 1777 e il v. 5 nel 1782). L'opera di Roselli fu stampata in prima edizione divisa in quattro parti, delle quali la seconda occupa tre distinti volumi; si può pertanto concepire l'opera sia come costituita da quattro volumi, di cui il secondo diviso in tre tomi, sia come costituita da sei volumi. Utilizzo la seconda modalità di descrizione dell'opera.

SACRA STUDIORUM CONGREGATIO. Theses quaedam, in doctrina sancti Thomae Aquinatis contentae, et a philosophiae magistris propositae, adprobantur. In: ACTA Apostolicae Sedis. Romae: Typis poliglottis Vaticanis, 1914. v. 6, p. 383-386.

SANTINELLO, Giovanni; PIAIA, Gregorio (a cura di). Storia delle storie generali della filosofia. Brescia-Padova: La Scuola-Antenore, 1979-2004. 5v. Edizione aggiornata in lingua inglese: Models of the History of Philosophy. Dortrecht: Kluwer-Springer, 1993-.

SIXTUS V. Bulla Thriumphantis Hierusalem. 1588. In: TOMASSETTI, Aloysi (ed.). Bullarium Romanum. Augustae Taurinorum: Seb. Franco et Henrico Dalmazzo editoribus, 1863. v. 8, p. 1005 b-1012b.

SPADARO, Antonio. Intervista a Papa Francesco. L'osservatore romano, 21 settembre 2013, p. 5-8.

THOMASIUS, Christianus. Introductio ad philosophiam aulicam seu primae lineae libri de prudentia cogitandi atque ratiocinandi ubi ostenditur media inter praejudicia Cartesianorum, et ineptias peripateticorum, veritatem inveniendi viam. Lipsiae: Apud autorem, 1688.

THOMASIUS, Jacobus. Theologia scholastica et eius initium. In: THOMASIUS, Christianus (ed.). Historia sapientiae et stultitiae. Halae Magdeburgicae: Typis et sumptibus Christopheri Salfeldii, [1693?]. v. 3, p. 225-243. 
FORLIVESI, M.

TOLOMIO, Ilario. Dimenticare l'antimodernismo: filosofia e cultura censoria nell'età di Pio X. Padova: CLEUP, 2007.

VIAN, Giovanni. Il modernismo: la Chiesa cattolica in conflitto con la modernità. Roma: Carocci, 2012.

ZAMBARBIERI, Annibale (a cura di). I cattolici e lo stato liberale nell'eta di Leone XIII. Venezia: Istituto Veneto di Scienze, Lettere ed Arti, 2008. 